\title{
Path Planning of Autonomous Underwater Vehicles for Adaptive Sampling Using Mixed Integer Linear Programming
}

\author{
Namik Kemal Yilmaz, Constantinos Evangelinos, Pierre F. J. Lermusiaux, and \\ Nicholas M. Patrikalakis, Member, IEEE
}

\begin{abstract}
The goal of adaptive sampling in the ocean is to predict the types and locations of additional ocean measurements that would be most useful to collect. Quantitatively, what is most useful is defined by an objective function and the goal is then to optimize this objective under the constraints of the available observing network. Examples of objectives are better oceanic understanding, to improve forecast quality, or to sample regions of high interest. This work provides a new path-planning scheme for the adaptive sampling problem. We define the path-planning problem in terms of an optimization framework and propose a method based on mixed integer linear programming (MILP). The mathematical goal is to find the vehicle path that maximizes the line integral of the uncertainty of field estimates along this path. Sampling this path can improve the accuracy of the field estimates the most. While achieving this objective, several constraints must be satisfied and are implemented. They relate to vehicle motion, intervehicle coordination, communication, collision avoidance, etc. The MILP formulation is quite powerful to handle different problem constraints and flexible enough to allow easy extensions of the problem. The formulation covers single- and multiple-vehicle cases as well as singleand multiple-day formulations. The need for a multiple-day formulation arises when the ocean sampling mission is optimized for several days ahead. We first introduce the details of the formulation, then elaborate on the objective function and constraints, and finally, present a varied set of examples to illustrate the applicability of the proposed method.
\end{abstract}

Index Terms-Adaptive sampling, Autonomous Ocean Sampling Network (AOSN), autonomous underwater vehicle (AUV), data assimilation, error subspace, mixed integer linear programming (MILP), Monterey Bay, ocean modeling, ocean observing system, path planning, routing, trajectory planning.

Manuscript received June 25, 2006; revised December 27, 2007; accepted June 25, 2008. Current version published February 06, 2009. This work was supported in part by the National Science Foundation under ITR Grant EIA-0121263 and the Department of Commerce under NOAA-MIT Sea Grant College Program Grant NA86RG0074. The work of P. F. J. Lermusiaux was supported by the U.S. Office of Naval Research under Grants N00014-05-1-0335, N00014-04-1-0534, N00014-05-G-0106, N00014-05-1-0370, and N00014-07-1-1061. The work of N. M. Patrikalakis was supported by the National Research Foundation of Singapore under the SMART Center CENSAM project.

Associate Editor: D. J. Stilwell.

N. K. Yilmaz is with Tigres Ltd. Co., Turgutlu/Manisa 45400, Turkey (e-mail: nkyilmaz@alum.mit.edu).

C. Evangelinos is with the Department of Earth, Atmospheric, and Planetary Sciences, Massachusetts Institute of Technology, Cambridge, MA 02139-43071 USA (e-mail: ce107@mit.edu).

P. F. J. Lermusiaux and N. M. Patrikalakis are with the Department of Mechanical Engineering, Massachusetts Institute of Technology, Cambridge, MA 02139-43071 USA (e-mail: pierrel@mit.edu; nmp@mit.edu).

Color versions of one or more of the figures in this paper are available online at http://ieeexplore.ieee.org.

Digital Object Identifier 10.1109/JOE.2008.2002105

\section{INTRODUCTION}

$\mathbf{R}$ EAL-TIME ocean forecasting is a challenging task due to issues that involve the intermittent nature of the ocean, the practical inability to make extensive and sustained in situ measurements, the uncertainties in the initial and boundary conditions, and the limited information at depth to complement the satellite measurements. To accurately forecast the evolution of a complex system as the ocean, one needs to take into account the possibly large deviations of the solution due to small initial and boundary uncertainties [1]-[4]. Weather and ocean forecasts also suffer from intrinsic uncertainties that arise due to errors in the model formulation and errors in its numerical solution. Finally, even if one could uniformly sample the ocean, much of the data corresponding to regions of low dynamical variability would be redundant while data pertaining to regions of high dynamical variability would be lacking resolution. Therefore, to utilize the measuring assets in an optimal way, one must plan ahead the sampling strategy to be followed. Our work describes, implements, illustrates, and evaluates new technical schemes for the optimal planning of the path of ocean platforms based on mixed integer linear programming (MILP) and advanced uncertainty estimates for ocean prediction.

Observation networks used for weather and ocean forecasting can be thought of being composed of a routine and an adaptive component [5]. The routine component comprises observations from the fixed observing network, satellite measurements, and other measurements that are routinely taken. The routine component collects the data that is situation independent. An additional component can be utilized to collect more data in regions critical to a specific objective. This objective is often a function of the synoptic oceanic or atmospheric dynamics and variability. The additional network component thus needs to be adaptive because the form of the objective can be modified but also because the fields to be measured are dynamic [6]. For example, for adaptive sampling on a daily time scale, the critical regions to be measured can be expected to vary from day to day. In the ocean, the adaptive component might involve ships or (un)manned aircrafts that drop instruments in data-sensitive regions, autonomous underwater vehicles (AUVs), gliders, etc. In an ocean estimation problem, measurements can impact both past and future field estimates as a function of advection, diffusion, and other ocean processes. The adaptive network can then be continually directed to locations that maximize the expected improvements in some aspect of the estimation. This problem 
is known as targeting, adaptive observations, or adaptive sampling [7]-[9].

Adaptive sampling can serve several purposes and has different forms. When scarcity of measurement assets exists, the whole routine component can also be treated as an adaptive network. Adaptive observation schemes have several goals, such as decreasing the uncertainty, gathering critical information about the dynamics of the system, increasing the coverage of the system, etc. An important goal is to increase the accuracy of the estimates of the states of interest by utilizing the resources at hand in an optimal manner. The estimates can be as follows: 1) the nowcast fields, e.g., determine the data needed now to best improve current estimates; 2) the forecast fields, e.g., determine the data needed before the target prediction that will best improve this prediction; or 3) the past fields, e.g., determine the data that will minimize errors in the initial conditions. Societal applications of adaptive sampling are given in [10].

A variety of techniques have been employed to determine the ideal location of extra observations within an adaptive sampling network. Because the goal is to combine data and models, most are based on data assimilation approaches [11]. These techniques include singular vector technique [12]-[15], the analysis sensitivity technique [16], the observations technique [17], the ensemble transform (ET) technique [3], the Kalman filter technique [18], the ensemble transform Kalman filter (ETKF) technique [5], [19], and the nonlinear error subspace statistical estimation (ESSE) technique [7], [20]-[22].

Although all these techniques are very useful in different ways to distinguish potential regions for extra observations, they do not intrinsically provide a path for the adaptive platforms. Path planning of the adaptive elements for the network is often performed based on predesigned tracks as explained, for example, in [19], [21], and [22]. As the size of the adaptive network grows, the complexity of the routing problem gets amplified and the lack of rule-based path-planning schemes can lead to suboptimal plans.

Although adaptive sampling is now becoming an active research area, rule-based high-level path planning for oceanic adaptive sampling has not yet received a lot of attention. Previous work in environmental path generation includes path planning for atmospheric networks [19], [14], which can have quite different considerations than an ocean network including assets such as AUVs and internal ocean dynamics at mesoscales that are usually slower than weather scales. In ocean adaptive sampling, the body of previous work involves low-level path planning, control, and coordination issues. The commonality of these approaches is that either waypoints are given a priori or that simple and local search techniques such as gradient methods (greedy search) are employed to locate the waypoints [23], [19]. The use of such local techniques is useful and promising, but it does not guarantee global optimality. Other ocean engineering schemes include redundant manipulator methods for partially decentralized path planning of several vehicles [24], [25].

Optimal sampling algorithms with similarities to ours have been used in other scientific and engineering domains, but often with different objectives, constraints, and types of asset behavior. Such algorithms and approaches include the selec- tive traveling salesman problem (STSP), routing problems, and some particular path-planning problems [26]-[28], [23], [29], [30]. In STSP, there are nodes with some award points associated to them. Given a limited travel time, the aim is to collect as much reward as possible. Unlike the classic traveling salesman problem (TSP), not all the nodes need to be visited. Only the most rewarding nodes are to be targeted. In the ocean, the existence of many geometrical and operational constraints, and the fact that the terminal location of the vehicle is unknown at the beginning of the problem, make the path generation problem remarkably different and more difficult than STSP. Recent research exists on path planning and coordination issues of unmanned aerial vehicles (UAVs) [29], [30], which present a good insight to the use of MILP in path planning. Collecting rewards along the paths of the vehicles in our case correspond to taking line integrals along each path. Another difference is the lack of waypoint information in our case.

In what follows, we first lay out the problem statement (Section II) and develop and determine the objective function (Section III). We then formulate a set of motion constraints (Section IV) and present a solution method for the optimum generation of the observational paths (Section V). We carry out a series of examples, with single time cases (Section VI) and multiple time situations (Section VII). We conclude with Section VIII.

\section{Problem Statement}

To carry out adaptive sampling, we first need a field that ranks and locates the regions of interest. These regions may be characterized by using the uncertainty predictions on the states [error variances, probability distribution fields (PDFs), etc.] or physical features of dynamical interest (eddies, upwelling, jets, etc.). The former is a vector or a scalar field (continuous in time and space) provided by the Harvard Ocean Prediction System (HOPS) and error subspace statistical estimation (ESSE) system [31]-[33], whereas the latter is a set of subregions that needs to be selected manually or directly detected using feature extraction [34], [35] and possibly presented as a Boolean field (e.g., discontinuous field). Because information from both sources is valuable, it is advantageous to combine the two sources of information. This involves the investigation of an optimal way to merge two different requirements into a single field [36]. In this study, it is assumed that such a combined field is given. The methods developed and implemented are independent of the type of fields. For the examples provided, the fields used are uncertainty information on ocean states provided by ESSE/HOPS, which we refer to as "uncertainty fields."

The uncertainty field is representative of the location of observation points to be targeted. For a nowcast, high uncertainty values correspond to the coordinates that are primarily worth visiting. The oceanographic assets that are generally available for sampling include buoys, autonomous surface crafts (ASCs), AUVs, gliders, and oceanographic ships, which can be utilized also to deploy the AUVs. In this study, we focus on the pathplanning problem for AUVs, which is sufficiently generic to also allow the solution of sampling problems with other available assets. 


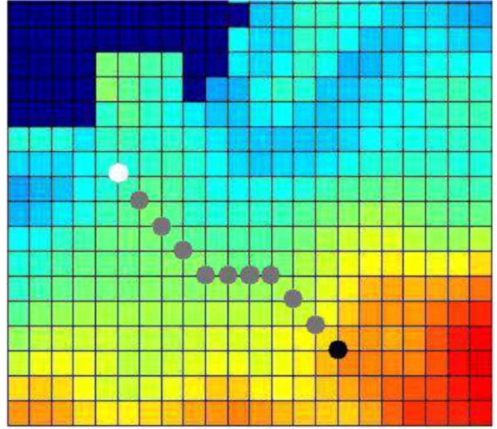

(a)

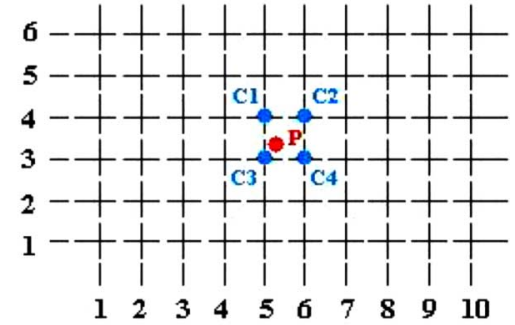

(b)

Fig. 1. (a) Path construction by segmentation. (b) Representation of a field value at any coordinate as a convex combination of the values of the four neighboring grid points.

The problem at hand is a constraint-optimization problem. The objective is to sample the regions of greatest uncertainty. For a group of assets (AUVs), it can be stated as finding the optimal sampling patterns/routes within the specified constraints (vehicle motion, intervehicle coordination, communication, collision avoidance) such that the total observational gain during the travel of the assets is maximized. By observational gain we mean the uncertainty values. The complete picture of the problem is obtained when multiple time scales are considered. A standard ocean approach to adaptive sampling has been to consider the nowcast problem and to construct the observational waypoints or paths on a day-by-day basis. For planning further ahead in time, one approach is to treat the optimization problem in an intertemporal manner. In generating an AUV path over two days, tomorrow's starting point is then related to today's endpoint. Instead of a myopic approach, coupling the paths belonging to consecutive days allows a more farsighted optimality. This requires, of course, that an estimate of the uncertainties on the state estimates is available for each day in our targeted time scale. In general, adaptive sampling paths can be planned for as far ahead in time as the time for which the fields of interest are predictable.

If the physical sampling optimized by the present approach is carried out, the data collected are utilized, either on their own or are assimilated in ocean models for optimal field estimation. This is what is being simulated in this paper. In all cases, the data or the data-driven model estimates can be utilized for scientific studies and societal applications. We refer, for example, to [4] and [11].

Inputs to the problem are here chosen to be the uncertainty fields and the unknowns in the problem are the $x$ and $y$ coordinates of the paths of each vehicle involved in the problem. The path of $p$ th vehicle is discretized into $N_{p}-1$ segments by using $N_{p}$ points and assigned variables that stand for $x$ and $y$ coordinates. Then, with the desired objective function and proper problem constraints, the optimizer is expected to solve for the $x$ and $y$ coordinates for each discrete waypoint. The path is constructed by connecting consecutively numbered points. The lower and upper limits on the $x$ and $y$ values are determined by the coordinates of the terrain under consideration. This framework is depicted in Fig. 1(a). The starting point of motion that is supplied as the initial condition to the system is shown by a white dot. The black dot denotes the terminal point, and the gray dots show the intermediate points.

\section{OBJeCTIVE FUNCTION}

In a 2-D discrete scenario for a single vehicle, our objective function can be written as

$$
\max \sum_{i=1}^{N} P(x(i), y(i))
$$

where $P$ stands for the 2-D array that represents the uncertainty field and $x(i)$ and $y(i)$ represent the $x$ and $y$ coordinates of the $i$ th point on the path. In the single-vehicle case, $x$ and $y$ happen to be vectors of length $N$. The starting point coordinates of the vehicle, which are represented by $x(1)$ and $y(1)$, are inputs to the problem. The remaining elements of the $x$ and $y$ vectors are unknowns we are trying to solve for. When we solve for $x$ and $y$, it means we determine the $x$ and $y$ coordinates of the vehicle's waypoints.

In general, the uncertainty fields we are dealing with are neither convex nor concave functions. This characteristic requires the use of piecewise curve fitting to properly represent the field. In our approach, we chose to use linear piecewise curve fitting. In an optimization formulation framework, this can be achieved by the use of special ordered set (SOS) of type 2. An SOS2 is a set of continuous or integer variables among which only two variables can be nonzero. Also, it is required that the two nonzero variables must have adjacent indices. Using SOS2 functionality, it is possible to approximate a nonconvex, nonconcave 2-D nonlinear function such as the objective function shown in (1). This concept was first introduced by Beale and Tomlin [37] and has been developed by Williams [38]. We refer the reader to [38] for a detailed discussion of the topic.

Let $U_{i j}$ denote the values of the function $z=f(x, y)$ on the computational 2-D grid (the grid spacing does not need to be equidistant). Any given function $z=f(x, y)$ can be approximated by the following:

$$
\begin{aligned}
& \sum_{i} \mathrm{DX}_{i} l x_{i} \text { is } \mathrm{SOS} 2 \\
& \sum_{j} \mathrm{DY}_{j} l y_{j} \text { is } \mathrm{SOS} 2
\end{aligned}
$$


where $l x$ and $l y$ are arrays that correspond to unknown weights at the integer $x$ and $y$ coordinates over which function $z=$ $f(x, y)$ is defined. DX and DY are arrays of integers that define the range of the $x$ and $y$ coordinates of the given $U_{i j}$, respectively. Their elements are equated to the corresponding index $\left(\mathrm{DX}_{i}=i, \mathrm{DY}_{j}=j\right)$. They are used to weave the grid region under consideration. When we define $\sum_{i} \mathrm{DX}_{i} l x_{i}$ to be SOS2, it means that, in the output set, only two adjacent element can be nonzero. We also define

$$
\begin{aligned}
& \sum_{j} l x y_{i j}=l x_{i} \quad \forall i \\
& \sum_{i} l x y_{i j}=l y_{j} \quad \forall j
\end{aligned}
$$

where $l x y$ is a weight matrix, $l x y_{i j}$ stands for the weight at a specific integer point coordinate and is a function of $l x$ and $l y$, and $l x, l y$, and $l x y$ are all unknown variables in the formulation. These "auxiliary" variables are dependent on the targeted $x, y$ values and will be determined accordingly. Equations (4) and (5) establish the conservation of weight along each row and column. Then, we introduce the requirements

$$
\begin{aligned}
& \sum_{i} l x_{i}=1 \\
& \sum_{j} l y_{j}=1 .
\end{aligned}
$$

Equations (6) and (7) enforce convexity. For a noninteger coordinate, this condition guarantees that the corresponding $z$ value will be a convex combination of that of two or four neighboring integer coordinates.

For example, for the field presented in Fig. 1(b), DX contains the values of $1,2 \ldots 10$ and DY contains the values $1,2 \ldots 6$. The first SOS 2 condition states that the multiplication of $l x$ with DX will create a set of numbers such that at most two elements in the set can be nonzero and they must be adjacent. If one calculates $f(3.5,5.5)$, this condition ensures that only the elements corresponding to $x=3$ and $x=4$ in the set should turn out to be nonzero. A similar argument is true for the $y$ coordinate. As a result, at most four elements in both directions might come out to be nonzero and the values of $U_{x y}$ corresponding to these four coordinates will be used to approximate the value of $f(x, y)$ at a noninteger coordinate.

Then, $x, y$, and $f$ can be calculated using

$$
\begin{aligned}
x & =\sum_{i} \mathrm{DX}_{i} l x_{i} \\
y & =\sum_{j} \mathrm{DY}_{j} l y_{j} \\
f & =\sum_{i} \sum_{j} U_{i j} l x y_{i j} .
\end{aligned}
$$

In conjunction with the other constraints added, the optimal values of $x$ and $y$ and the resulting value of function $f$ will be fixed to satisfy the given objective function.

The above discussion lays out the foundation for representing the nonlinear field and the above formulation is good for finding the value at a single $(x, y)$ coordinate. In our problem, however, the path is segmented by $N$ points and the above formulation should be carried out at each path point. In the case of multiple vehicles, every path point belonging to each vehicle needs to be accounted for. For these extensions to the multiple-vehicle and multicoordinate case, one simply adds additional indices to the notation introduced above. The reader can refer to [39] for further details.

The objective is to maximize the summation of line integrals of the uncertainty values along the path of each vehicle in the fleet. The objective function can be written as

$$
\max \sum_{p=1}^{P} \sum_{k=1}^{N_{p}} f_{p k}
$$

where $P$ is the total number of vehicles in the fleet and $N_{p}$ is the total number of path points belonging to the $p$ th vehicle. In all of the above equations, subscripts $p$ and $k$ stand to denote the $k$ th path point of $p$ th vehicle. $U_{i j}$ (which is composed of the discretized values of the function $z=f(x, y)$ on the computational 2-D grid) stands for the uncertainty field data, which is the input to our problem.

\section{Motion CONSTRAints}

For the vehicles to move in a desired manner, some constraints that shape the vehicle navigation are needed: primary motion, anticurling, vicinity, communications, and obstacle avoidance constraints.

\section{A. Primary Motion Constraints}

When the vehicle is at a particular path point except the terminal point, it needs to move to the next path point. A constraint is thus needed to thrust the vehicle to the next path point. Referring to Fig. 2(a), if the vehicle is at the point numbered "1," it can only move to one of the eight adjacent points labeled "Y."

Explained motion can be achieved by the following set of constraints:

$$
\begin{aligned}
& \text { - } \forall p \in[1, \ldots, P] \text { and } \forall i \in\left[2, \ldots, N_{p}\right] \\
& x_{p i}=x_{p(i-1)}+b_{p i 1}-b_{p i 2} \\
& b_{p i 1}+b_{p i 2} \leq 1 \\
& y_{p i}=y_{p(i-1)}+b_{p i 3}-b_{p i 4} \\
& b_{p i 3}+b_{p i 4} \leq 1 \text {. } \\
& b_{p i 1}+b_{p i 2}+b_{p i 3}+b_{p i 4} \geq 1 . \\
& \text { - } \forall p \in[1, \ldots, P], \forall i \in\left[2, \ldots, N_{p}\right] \text {, and } \forall j \in[1, \ldots, 4] \\
& b_{p i j} \in 0,1 \text {. }
\end{aligned}
$$

The $b_{p i 1}, b_{p i 2}, b_{p i 3}$, and $b_{p i 4}$ in (12)-(17) are auxiliary binary variables needed to model the propulsive motion constraint. If $b_{p i 1}$ is set to 1 and $b_{p i 2}$ is set to 0 , then the $x$ coordinate of the next path point will be one unit greater than that of the current one and similarly for the $y$ coordinate. If they are both set to zero, it means that the $x$ coordinate should not change, which is 


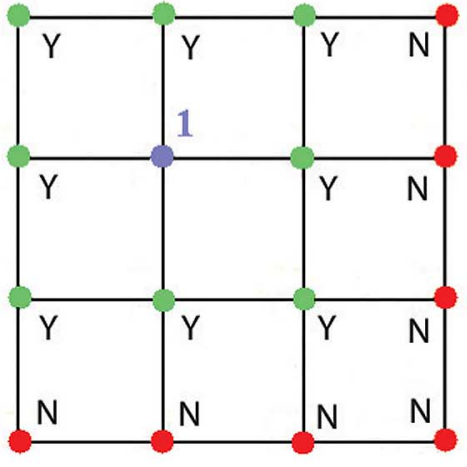

(a)
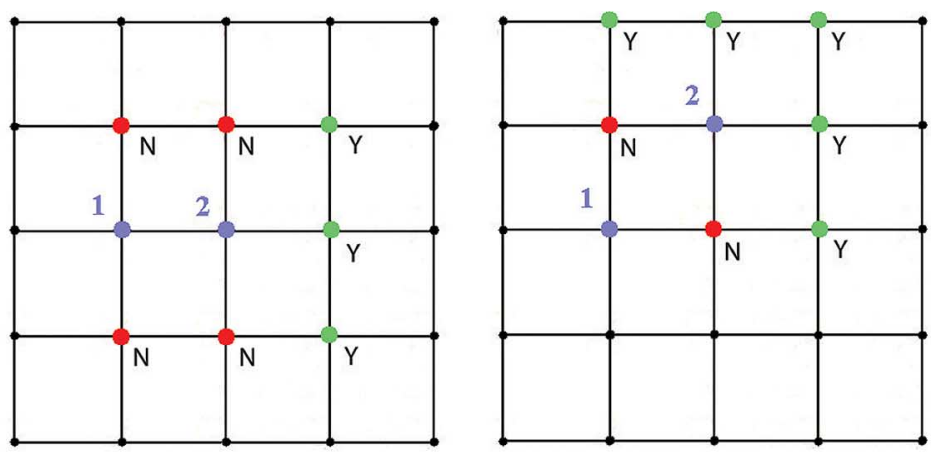

(b)

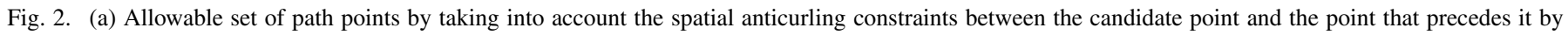

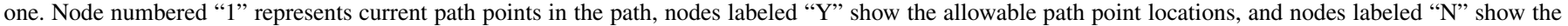

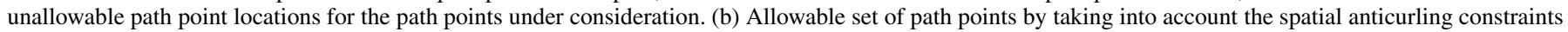


the allowable path point locations, and nodes labeled "N" show the unallowable path point locations for the path points under consideration.

an allowable possibility. Another scenario that will keep the $x$ coordinate fixed is when both $b_{p i 1}$ and $b_{p i 2}$ are set to 1 . This is a degenerate case and it must be avoided. To ensure both $b_{p i 1}$ and $b_{p i 2}$ will not be set to 1 at the same time, (13) is introduced. A similar argument follows for the $y$ coordinate; see (14) and (15). To avoid the vehicle get stuck at the same $x$ and $y$ coordinates, (16) is included.

\section{B. Anticurling/Winding Constraints}

When a point in the map is visited and measurements are made, not only the uncertainty value at the particular point but also the uncertainty value at the neighboring points is decreased, due to correlations among ocean values. Therefore, there exists an area of influence for a measurement. If the vehicle curls around the same area too much, it will be visiting points whose uncertainty values are already decreased by previous measurements. This results in inefficient consumption of range. To introduce correlations along the vehicle paths, we relate the coordinates of a path point to these of the path point preceding it by two path points, to the coordinates of the path point preceding it by three path points, and so on up to the desired point depending on a given range value. The parameters in this approach can adjust the straightness/curvature of the path, as desired.

The first set of constraints that will be imposed to straighten the path involves the relative location of the $x$ and $y$ coordinates of a path point with respect to the $x$ and $y$ coordinates of the path point that precedes it by two. These constraints can be described as follows:

$$
\begin{aligned}
& \text { - } \forall p \in[1, \ldots, P] \text { and } \forall i \in\left[3, \ldots, N_{p}\right] \\
& \left|x_{p i}-x_{p(i-2)}\right| \geq \Delta_{1} \text { OR }\left|y_{p i}-y_{p(i-2)}\right| \geq \Delta_{1} .
\end{aligned}
$$

In this formulation, $\Delta_{1}$ is a design parameter and the choice of $\Delta_{1}$ should be made on a case-by-case basis. In our particular ocean simulation, we have chosen $\Delta_{1}$ as two grid points, which physically corresponds to $3 \mathrm{~km}$. This is a good choice for the numerical resolution, field correlations, and asset ranges we deal with in this simulation. Fig. 2(b) depicts some allowable move scenarios. Blue dots numbered as " 1 " and " 2 " are the two preceding path points. The nodes labeled " $N$ " show the unallowable moves. The nodes labeled "Y" show the allowable locations for the next path points. These choices of allowable path points are generated by aggregately taking into account the previous propulsive constraints.

However, (18) is a nonlinear constraint and must be transformed into a linear one to be used in an MILP. This transformation will be done in two steps. First, the absolute value constraint can be eliminated by the following transformation:

- $\forall p \in[1, \ldots, P]$ and $\forall i \in\left[3, \ldots, N_{p}\right]$

$$
x_{p i}-x_{p(i-2)} \geq \Delta_{1}
$$

or

$$
x_{p(i-2)}-x_{p i} \geq \Delta_{1}
$$

or

$$
y_{p i}-y_{p(i-2)} \geq \Delta_{1}
$$

or

$$
y_{p(i-2)}-y_{p i} \geq \Delta_{1} \text {. }
$$

Equation (19) is a disjunctive constraint and is not a suitable kind of constraint for an MILP formulation. Constraints in an MILP formulation must be conjunctive. It is possible to transform a disjunctive constraint into a conjunctive one by using auxiliary binary variables and "Big-M" constants [40]. Such transformation yields the following:

- $\forall p \in[1, \ldots, P]$ and $\forall i \in\left[3, \ldots, N_{p}\right]$

$$
x_{p i}-x_{p(i-2)} \geq \Delta_{1}-M t 1_{p i 1}
$$

and

and

$$
x_{p(i-2)}-x_{p i} \geq \Delta_{1}-M t 1_{p i 2}
$$

and

and

$$
y_{p i}-y_{p(i-2)} \geq \Delta_{1}-M t 1_{p i 3}
$$

$$
y_{p(i-2)}-y_{p i} \geq \Delta_{1}-M t 1_{p i 4}
$$




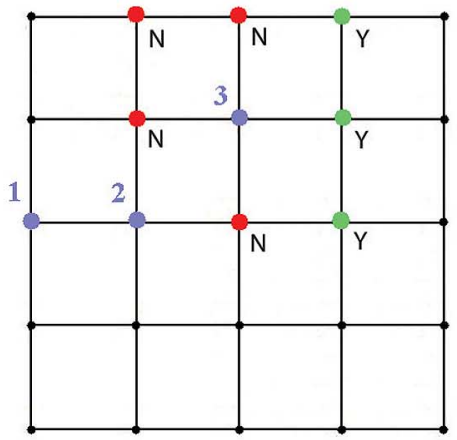

(a)



a)

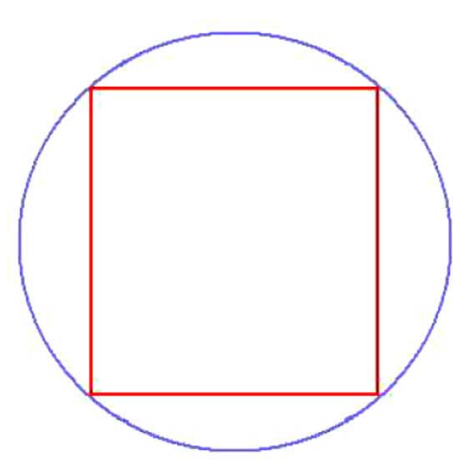

(b)

Fig. 3. (a) Allowable set of path points by taking into account the spatial anticurling constraints between the candidate point and the point that precedes it by two. Nodes numbered "1," "2," and "3" represent current path points in the path, nodes labeled "Y" show the allowable path point locations, and the nodes labeled "N" show the unallowable path point locations for the path points under consideration. (b) Approximation of a circle by a square.

and

$$
\begin{aligned}
\sum_{w=1}^{4} t 1_{\text {piw }} & \leq 3 \\
t 1_{\text {piw }} & \in 0,1 \quad \forall w \in[1, \ldots, 4] .
\end{aligned}
$$

In the above formulation, $M$ is a number safely bigger than any of the numbers that may appear on any side of the inequalities.

As a variation of the same theme, the second set of constraints that will be imposed to straighten the path involves the relative location of the $x$ and $y$ coordinates of a path point with respect to the $x$ and $y$ coordinates of the path point that precedes it by two. These constraints can be written as follows:

$$
\begin{aligned}
& \text { - } \forall p \in[1, \ldots, P] \text { and } \forall i \in\left[4, \ldots, N_{p}\right] \\
& \left|x_{p i}-x_{p(i-3)}\right| \geq \Delta_{2} \text { OR }\left|y_{p i}-y_{p(i-3)}\right| \geq \Delta_{2} .
\end{aligned}
$$

For our simulation, we choose $\Delta_{2}$ to be 2.5 grid units, which physically corresponds to $3.7 \mathrm{~km}$. Fig. 3(a) shows some allowable move scenarios. As before, the constraint presented by (23) is combined with the other motion constraints: the allowable moves presented in Fig. 3(a) are a result of the collective restrictions on the navigation of the vehicle. The dots numbered as 1 , 2 , and 3 are the three preceding path points. Equation (23) can be transformed into an MILP formulation as explained above. The reader can see [39] for further details.

This approach can be extended to include the relation between more path points in a row to avoid curling that depends on the range of the vehicle and the features of a given field. The values of $\Delta_{1}$ and $\Delta_{2}$ can be estimated from the value of allowed minimum curvature. A good rule of thumb would be to choose $\Delta_{1}$ and $\Delta_{2}$ such that their average be equal to the allowed minimum curvature.

\section{Vicinity Constraints for Multiple-Vehicle Case}

In the case in which there exist multiple vehicles navigating to different regions of the mission zone, first and foremost, collisions between vehicles must be avoided. In any case, as discussed for the curling constraint, it is also disadvantageous for two vehicles to navigate too close to each other, even if they do not run the risk of colliding. In the case of multiple peaks in the uncertainty field and available vehicles starting their motion close to the stronger peak, the vicinity constraints can help vehicles get separated. Antivicinity constraint can thus, as a result, lead to the visits of some weaker peaks further away, which would not be visited otherwise.

To achieve this, for every pair of vehicles $p$ and $q$, every pair of path points must be a safe distance apart from each other. Let the safety distances in $x$ and $y$ directions be denoted by $\Delta x_{\text {safety }}$ and $\Delta y_{\text {safety }}$, respectively. The vicinity constraints then can be constructed as follows:

$$
\begin{aligned}
& \text { - } \forall p, q \in[1, \ldots, P]: \forall p, q \mid p>q \text { and } \forall i, j \in\left[1, \ldots, N_{p}\right] \\
& \left|x_{p i}-x_{q j}\right| \geq \Delta x_{\text {safety }} \text { OR }\left|y_{p i}-y_{q j}\right| \geq \Delta y_{\text {safety }}
\end{aligned}
$$

Utilizing the transformations to handle the absolute value and the conjunctive constraints as elucidated in Section IV-B, (24) can be expanded into an MILP formulation. The reader can refer to [39] for further details. Again, $\Delta x_{\text {safety }}$ and $\Delta y_{\text {safety }}$ are design parameters and can be varied depending on the field features, number of vehicles, and vehicle range.

\section{Coordination Issues Related to Communication With AUV}

There exist few different scenarios related to the communication issues and they are discussed below: AUV-ship, AUVshore, and AUV-network communications [41]-[43].

1) Coordination With a Ship and Ship Shadowing: The nature of our adaptive sampling problem can still often utilize a ship to move with the AUV fleet, so as to facilitate at-sea launch and retrieval of AUVs as well as fast transit and AUV battery recharge. In general, the AUV can communicate with the ship for data transmission. Inclusion of a ship in the adaptive network enables to visit locations far from the shore stations and perform broad area coverage problems [23]. The ship AUV coordination issue adds another dimension to the problem. The ship and the AUVs in the fleet that are linked to the ship must navigate in harmony to finish the mission successfully. When the mode of communication is brought into consideration, there emerge two main cases to consider. The first one is communication via acoustical means and the second is radio communication or direct link. 
Acoustical Communication: For an acoustical link to work [44], [45] during the mission, the AUV must stay near the ship. In addition, when its charge is consumed, it must either return to the ship or park at a surface location that is close enough to be picked up by a boat dispatched from the ship. To impose this characteristic to the AUV motion, we need to add some extra constraints. The endpoint of the AUV can either be specified to be the coordinate of the ship, or stay in the same vicinity of the vehicle as the rest of the path points, or it can be dictated that although it does not need to be the same as the ship coordinates (meaning returned to the ship) it should be closer to the ship than the rest of the path points. Also, to synchronize the motion of the ship and the AUV fleet, the path of the ship must be known. Once the ship path is known (ship path can be determined using ideas and methods similar to the ones presented in this paper), it can be segmented into as many path points as the AUV. Then, the time domain dependence is unique between identically indexed ship and AUV path points. The $n$th path point of the ship then corresponds to the location of the ship when the AUV visits its $n$th path point. If all AUVs in the fleet have the same number of path points, then a single segmentation is enough. If they do not have the same number of path points, then the ship path segmentation must be performed for every AUV. Assuming that the terminal path point of the AUV stays within the same distance to the ship as the other path points, these ideas can be put into MILP formulation as follows:

- $\forall p \in[1, \ldots, P]$ and $\forall i \in\left[1, \ldots, N_{p}\right]$

$$
\begin{gathered}
\left|x_{p i}-\operatorname{ship} \__{p i}\right| \leq \Delta x_{\text {ship_vicinity }} \\
\text { AND } \\
\mid y_{p i}-\text { ship_}_{y_{p i}} \mid \leq \Delta y_{\text {ship_vicinity }}
\end{gathered}
$$

where ship $\_x_{p i}$ and ship $\_y_{p i}$ stand for $x$ and $y$ coordinates of the $i$ th path point of the ship path segmentation for $p$ th vehicle. $\Delta x_{\text {ship_vicinity }}$ and $\Delta y_{\text {ship_vicinity }}$ are the constants that are used to define region of vicinity for a ship. The region of coverage can be thought of as a circle centered at the coordinate of the ship. Equation (25) approximates this circular region by a square for the sake of simplicity of formulation. This is shown in Fig. 3(b).

A more complex alternative to define this region is to approximate a circle by the biggest polygon that will fit inside the circle and write down the equations of lines that construct the polygon with $q$ edges as a function of coordinates of the $i$ th path point of $p$ th vehicle. The constraint set is formed by adding inequalities either of type $a_{p i q} x+b_{p i q} y \leq c_{p i q}$ or of type $a_{p i q} x+b_{p i q} y \geq c_{p i q}$, depending on the equation under consideration. This imposes the confinement of the $(x, y)$ point inside the hexagon. As explained before, (25) can again be transformed into an MILP. The reader can refer to [39] for further details.

a) Another issue that arises in ship AUV coordination is the collision avoidance between the AUVs and the ship. This condition can be met by introducing a minimum safety distance between the ship and the AUVs that must be observed during sampling. Extending the previous idea, this condition can be formulated as follows:

$$
\begin{gathered}
\cdot \forall p \in[1, \ldots, P] \text { and } \forall i \in\left[1, \ldots, N_{p}\right] \\
\left|x_{p i}-\operatorname{ship\_ } x_{p i}\right| \geq \Delta x_{\text {ship_safety }} \\
\text { OR } \\
\left|y_{p i}-\operatorname{ship\_ } y_{p i}\right| \geq \Delta y_{\text {ship_safety }} .
\end{gathered}
$$

The above equation can be transformed into an MILP formulation as explained above. The reader can refer to [39] for further details.

b) To handle the cases where the terminal path must be in a tighter vicinity of the vehicle or the AUV must return to the ship, the constraints that account for terminal path point must be specially treated. If an AUV is to return to the ship, we can have the extra constraint

$$
\begin{array}{ll}
x_{p N_{p}}=\operatorname{ship} \_x_{p N_{p}} & \forall p \in[1, \ldots, P] \\
y_{p N_{p}}=\text { ship_- } y_{p N_{p}} & \forall p \in[1, \ldots, P]
\end{array}
$$

c) Or if the terminal path point needs to lie in a tighter vicinity than the other path points for the ease of picking up, then we need to add the following constraints:

$$
\begin{gathered}
\cdot \forall p \in[1, \ldots, P] \\
\left|x_{p N_{p}}-\operatorname{ship\_ } x_{p N_{p}}\right| \leq \Delta x_{\text {ship_vicinity_TP }} \\
\text { AND } \\
\mid y_{p N_{p}}-\text { ship_- } y_{p N_{p}} \mid \leq \Delta y_{\text {ship_vicinity_TP }}
\end{gathered}
$$

where $\Delta x_{\text {ship_vicinity_TP }}$ and $\Delta y_{\text {ship_vicinity_TP }}$ stand for the tighter bounds in the vicinity of terminal path points to the ship. The above equation can be transformed into an MILP formulation as explained above. The reader can refer to [39] for further details.

Radio and Direct Communications: As aforementioned, the two other alternatives for communication are the radio link and the direct communication. If the preferred way of communication is opted to be wireless communication (radio), the AUV needs to be in some vicinity of the ship at the end of its motion to communicate with the ship. In that case, only (29) needs to apply. If direct connection is the selected communication method, the AUV needs to board the ship at the end of the mission, in which case, only (27) needs to be applied.

2) Communication With a Shore Station: In the case of shore station, the end path point coordinates of the vehicles need to either lie near the station location to establish radio communication or they must match with the coordinates of the shore station if they are required to return to it. If the vehicles need to lie in a proximity of the shore station to be picked up by a boat, we need to introduce the following constraints:

- $\forall p \in[1, \ldots, P]$

$$
\begin{gathered}
\mid x_{p N_{p}}-\text { shore } \_x \mid \leq \Delta x_{\text {shore_vicinity }} \\
\text { AND } \\
\mid y_{p N_{p}}-\text { shore_} y \mid \leq \Delta y_{\text {shore_vicinity }} .
\end{gathered}
$$




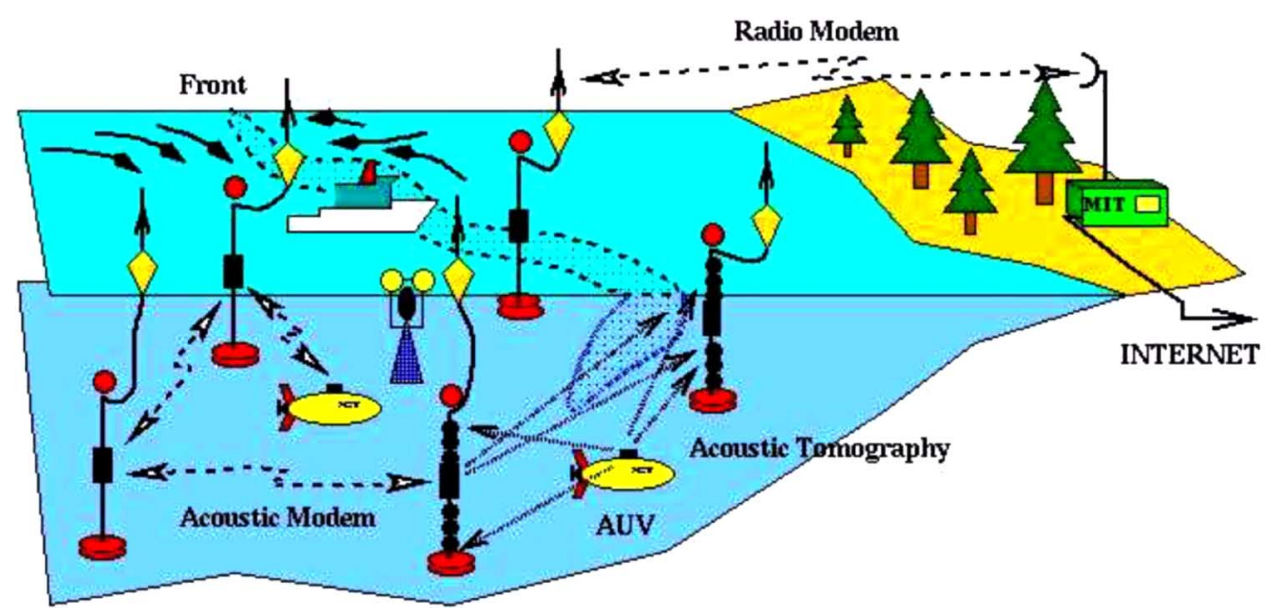

Fig. 4. Illustration of an Autonomous Ocean Sampling Network (AOSN) [47], [48].

where shore $\_x$ and shore $-y$ stand for the $x$ and $y$ coordinates of the shore station. Or, if the vehicle needs to return to the shore station, one can impose

$$
\begin{array}{ll}
x_{p N_{p}}=\text { shore } \_x & \forall p \in[1, \ldots, P] \\
y_{p N_{p}}=\text { shore } \_y & \forall p \in[1, \ldots, P] .
\end{array}
$$

3) Communication With an AOSN: The communication with buoys comes into play in the context of an Autonomous Ocean Sampling Network (AOSN), ${ }^{1}$ [46]. AOSN research is still very active. The main goal is to realize a completely autonomous network that efficiently collects data from the ocean. The network consist of AUVs, buoys, shore stations, acoustic modems, satellite and radio links, and any other potential autonomous vehicles such as gliders. In one scenario, the shore station makes the mission plan and sends it to the buoys via a radio link. Buoys establish an acoustical communication link with AUVs and upload the individual path plans to the AUVs. The AUVs navigate in accordance to the uploaded plan and make necessary measurements. When the mission is over, the collected data is transmitted to one of the buoys from which data is sent to the shore station using the wireless connection. Also, buoys not only act as an intermediate data logger but also as docking stations where AUVs can be recharged and continue their mission without the need to be carried to shore or to a ship. Fig. 4 illustrates an AOSN.

Even though a few large scale projects on AOSN have occurred or are underway, a complete and efficient physical implementation of a truly autonomous AOSN has not yet been realized. For example, a missing component has been a fully automated and sustained path planning. Nonetheless, we can still offer a formulation hinging around an extended functionality of buoys as docking stations in addition to being a node in the communication network. If we consider a single-day mission, we can introduce the condition that the AUV must return to the closest buoy at the end of the day and if we assume that we have $M$ buoys whose coordinates are represented by the arrays buoy_$x_{h}$ and buoy_$y_{h}$, we can write the following:

${ }^{1}$ http://www.mbari.org/aosn/default.htm
1) $\forall p \in[1, \ldots, P]$

$$
\begin{aligned}
x_{p N_{p}} & =\sum_{h=1}^{M} \text { buoy_}_{\_} x_{h} b v_{p h} \\
y_{p N_{p}} & =\sum_{h=1}^{M} \text { buoy_}_{-} y_{h} b v_{p h} \\
\sum_{h=1}^{M} b v_{p h} & =1 \quad \forall p \in[1, \ldots, P] \\
b v_{p h} & \in 0,1 \quad \forall h \in[1, \ldots, M] .
\end{aligned}
$$

Variables $b v_{p h}$ are auxiliary variables that help to choose one of the buoy coordinates as the endpoint coordinate of AUVs. Equation (32) guarantees that only one buoy coordinate will be assigned to a specific AUV. Also, depending on the docking capabilities of a buoy, we can impose the constraint that at most one AUV can park at a given buoy. This can be formulated as

$$
\sum_{p=1}^{N} b v_{p h} \leq 1 \quad \forall h \in[1, \ldots, M] .
$$

Other constraints related to the communication with buoys or some other constraint that cannot be foreseen at this time without an actual implementation of an AOSN might need to be added. Given the flexibility and strength of the suggested formulation framework, other requirements that could emerge depending on the specific implementation of an AOSN can easily be added to the formulation.

\section{E. Obstacle Avoidance}

In the case of existence of obstacles in the region of interest, the task of collision prevention with obstacles could be managed in two alternative ways. One option is to introduce inequalities that will remove the regions where obstacles lie from the feasible coordinate set of the vehicle navigation. Another, simpler approach is to set the uncertainty values within the regions occupied by the obstacles to a very large negative number. Those points will not be included in the solution because their 


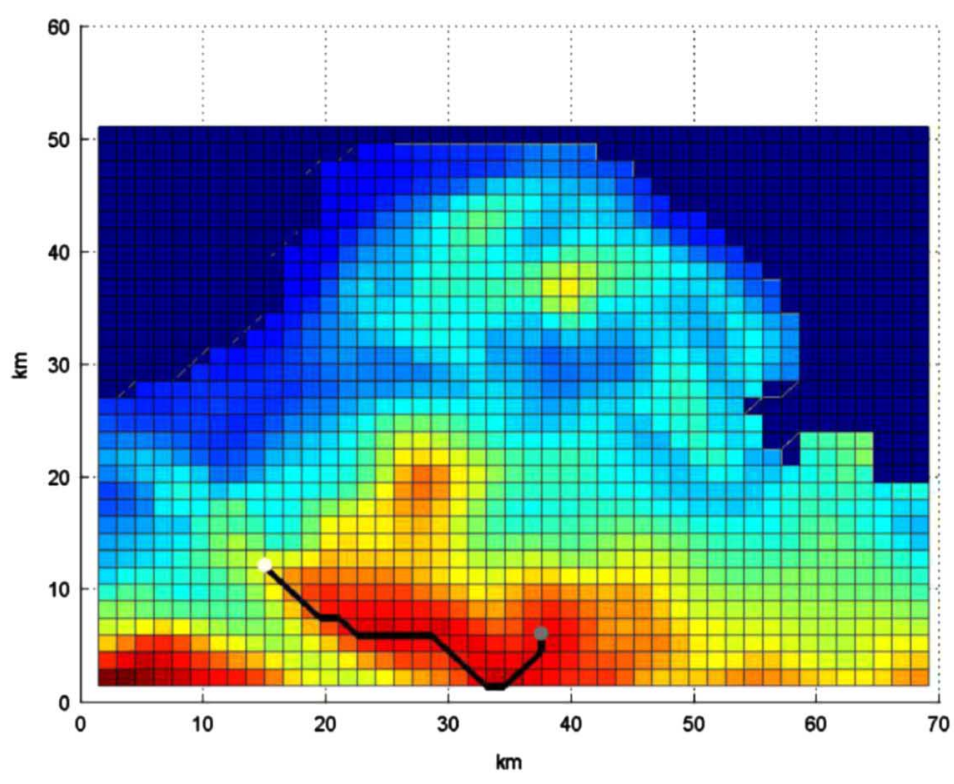

(a)

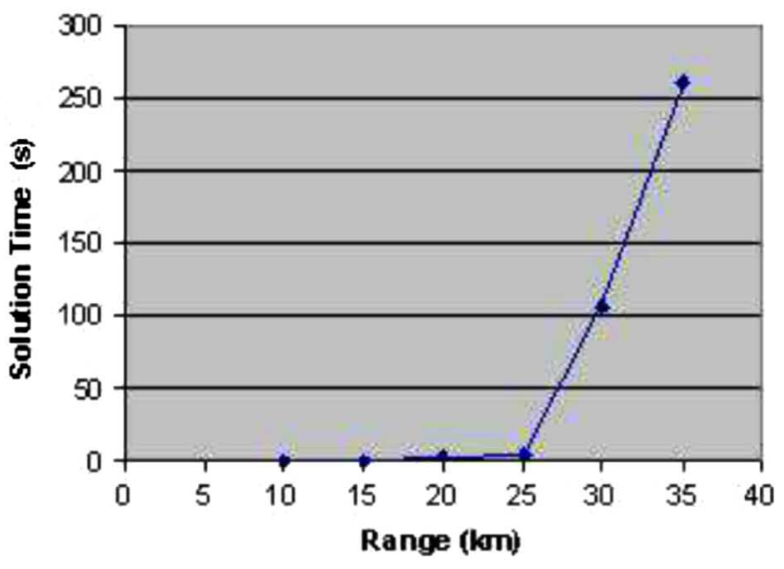

(b)

Fig. 5. (a) Results for the single-vehicle case. Starting coordinates and range: $x=15 \mathrm{~km}, y=12 \mathrm{~km}$, range $=30 \mathrm{~km}$, total reward $=1489{ }^{\circ} \mathrm{C}$. (b) Solution times for a single vehicle with starting position at $x=15 \mathrm{~km}$ and $y=12 \mathrm{~km}$.

inclusion will have negative contribution to the objective function. For other approaches on obstacle avoidance, we refer to [49]-[52].

\section{Methodology and Software Selection FOR THE MILP SOLUTION}

Our choice of implementation platform is the XPress-MP optimization package from "Dash Optimization."2 It has an MILP solver that uses brand and bound algorithm. It is suitable for solving our path-planning problem.

For optimum performance and ease of development, the ideal is to use a high-level modeling language that is compatible with the solver. Such modeling languages are especially made for optimization problems and are equipped with powerful tools to implement optimization problems faster. A modeling language offered by Dash Optimization is Mosel. Implementing the mathematical program in Mosel is straightforward, requiring minimal translation from the canonical form shown in (2)-(34). Also, Mosel is capable of easily implementing the SOS2 constraints.

\section{Results For Single-DAy CASE}

Our methodology and software have been tried on a wide variety of different scenarios with multiple types of fleet sizes, ranges, starting points, and constraints. The ocean fields that have been used are the temperature forecast uncertainty maps in Monterey Bay during August-September 2003 [22], [53], [54], as calculated by HOPS and ESSE systems (see Section II). In the examples that follow, most examples shown are for August 27, 2003, and they utilize uncertainty averages from the upper $(0-40 \mathrm{~m})$ ocean layers, focusing on the largest uncertainties in the ocean surface mixed layer and ocean thermocline dynamics. Depending on the objective, velocity or salinity fields

\footnotetext{
${ }^{2}$ http://www.dashoptimization.com
}

(or even a weighted average of all fields) can also be used with our software.

In all of the following graphs, gray dots indicate the starting point of the motion and white dots indicate the final point on the path. One important parameter in the problem formulation that controls the range for a given vehicle is the number of path points " $N_{p}$." It is not directly equal to the range because diagonal moves are allowed. Its value must be chosen based on the allowable range for each AUV on a given day. Once the problem is solved with the initial selection for " $N_{p}$," depending on the length of the generated path, some iterations might be necessary.

\section{A. Results for Single-Vehicle Case}

In this section, we look at an example where we have a single vehicle. Fig. 5(a) presents the path generated for a vehicle, given the starting coordinates and number of path points as the input along with the uncertainty field. As it can be seen on Fig. 5(a), the generated path successfully covers the regions of high uncertainty. To illustrate the solution time as a function of range, the same problem is solved for different path ranges. The result is presented in Fig. 5(b), which reveals that the solution time increases exponentially as a function of vehicle range. This behavior is expected because the formulation is a binary integer program and binary integer programs are known to be NP hard.

\section{B. Results for Multiple-Vehicle Case}

Often, multiple vehicles are available, which enables more thorough data collection in the region. It also brings some coordination issues such as avoiding vehicle collision and intelligent coordination of vehicles to cover as much critical regions as possible. The collision avoidance and coordination issues were handled by the introduction of (24). Fig. 6(a)-(c) shows paths generated for a two-vehicle case scenario as the vehicle path is gradually increased. As it can be observed from these figures, 


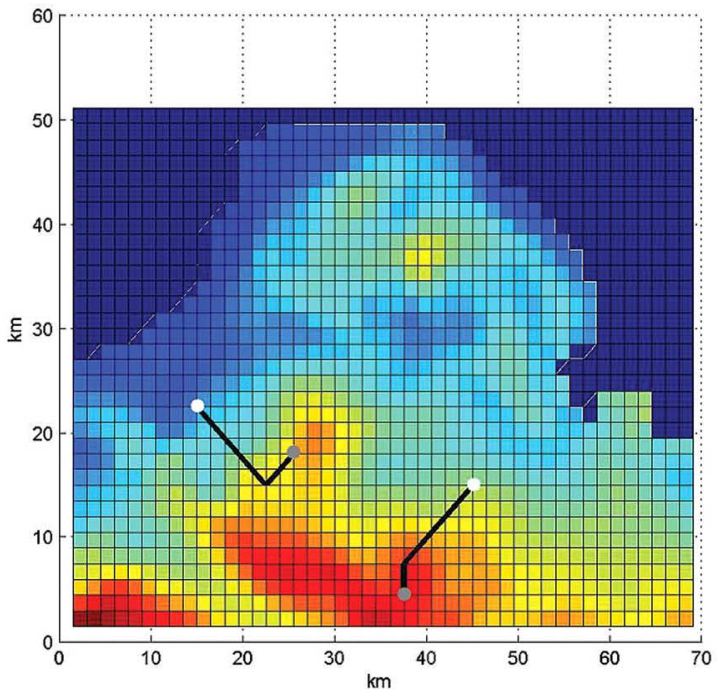

(a)

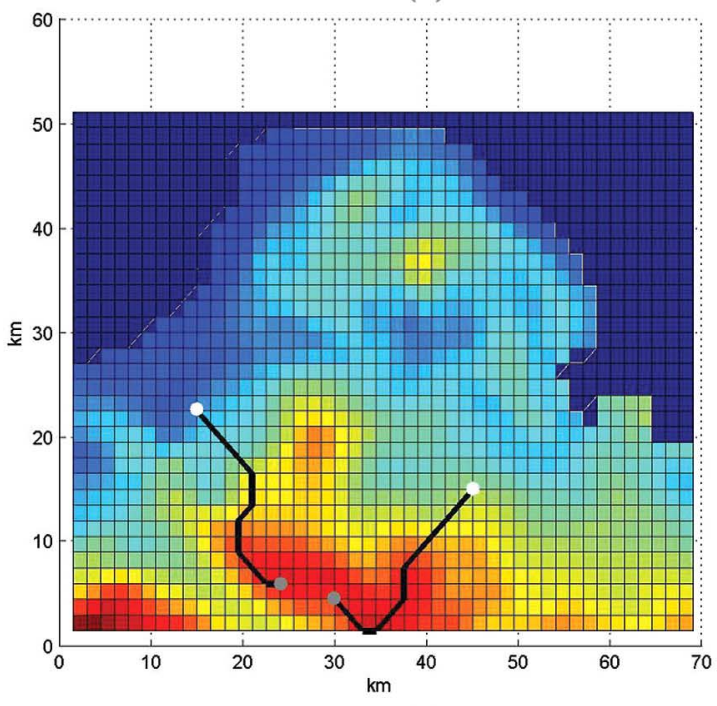

(c)

Fig. 6. (a) Results for the two-vehicle case. Starting coordinates and ranges: $x_{1}=15 \mathrm{~km}, y_{1}=22.5 \mathrm{~km}$, range ${ }_{1}=15 \mathrm{~km} ; x_{2}=45 \mathrm{~km}, y_{2}=15 \mathrm{~km}$, range ${ }_{2}=14 \mathrm{~km}$, total reward $=972{ }^{\circ} \mathrm{C}$. (b) Results for the two-vehicle case. Starting coordinates and ranges: $x_{1}=15 \mathrm{~km}, y_{1}=22.5 \mathrm{~km}$, range ${ }_{1}=16.5 \mathrm{~km} ; x_{2}=$ $45 \mathrm{~km}, y_{2}=15 \mathrm{~km}$, range ${ }_{2}=18 \mathrm{~km}$, total reward $=1273^{\circ} \mathrm{C}$. (c) Results for the two-vehicle case. Starting coordinates and ranges: $x_{1}=15 \mathrm{~km}, y_{1}=22.5 \mathrm{~km}$, range ${ }_{1}=22.5 \mathrm{~km} ; x_{2}=45 \mathrm{~km}$ and $y_{2}=15 \mathrm{~km}$, range ${ }_{2}=24 \mathrm{~km}$, total reward $=1879^{\circ} \mathrm{C}$. (d) Solution times for two vehicles with starting positions $x_{1}=$ $15 \mathrm{~km}, y_{1}=22.5 \mathrm{~km} ; x_{2}=45 \mathrm{~km}, y_{2}=15 \mathrm{~km}$.

the high-uncertainty regions are efficiently covered. The solution times are presented in Fig. 6(d), which again increases exponentially as a function of path points.

\section{Sensitivity to the Number of Vehicles}

The aim of this section is to show the sensitivity of the solution time to the number of vehicles involved in the path-planning task. We start with one vehicle and at each step introduce another vehicle until we reach five vehicles. Of course, each time a vehicle is added, a new global MILP optimization is carried out for all vehicles present. The paths of all vehicles are optimized at once. The results are presented in Fig. 7(a)-(e). The solution times are presented in Fig. 7(f). There is a sudden increase as the number of vehicles increases. This behavior is in agreement with the exponential complexity of the problem. Note that in
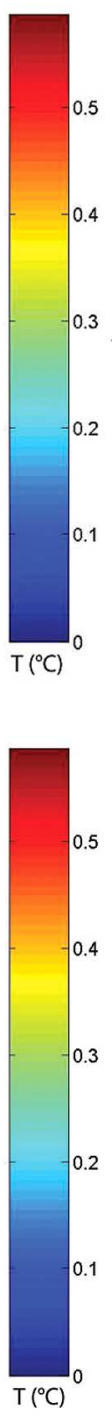

(d)

(b)
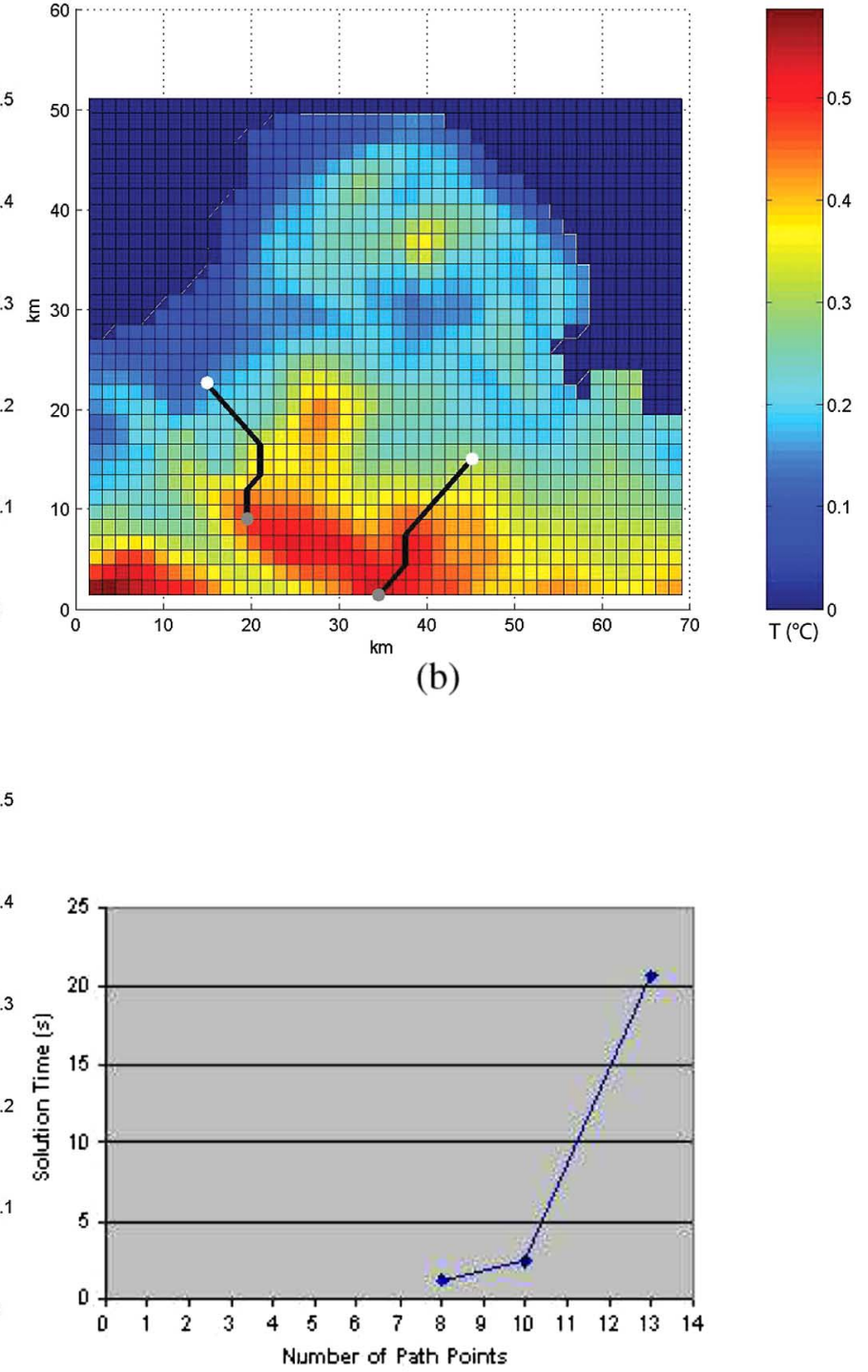

$15 \mathrm{~km}$, range
$6.5 \mathrm{~km} ; x_{2}=$
$y_{1}=22.5 \mathrm{~km}$,
ositions $x_{1}=$

this illustration of the sensitivity to the number of vehicles, the starting points of vehicles are selected far apart from each other deliberately so that the addition of another vehicle does not affect the previous solution by much. This peculiar behavior allows more direct comparisons with previous illustrations.

\section{Results With Ship Shadowing}

As explained earlier, during a mission, AUVs are generally accompanied by a ship. The AUVs are dropped from the ship for their mission and dock to, or are collected by, the ship at the end. To handle this situation, extra constraints are added to the formulation. The constraints to be used depend on the type of communication (see Section 4-D).

Fig. 8(a)-(b) shows two cases where the preferred mode of communication is chosen to be acoustical. Equations (25) and 


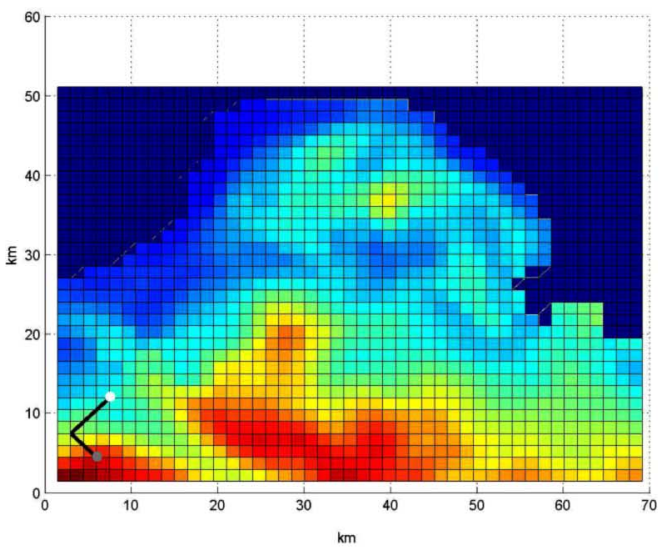

(a)

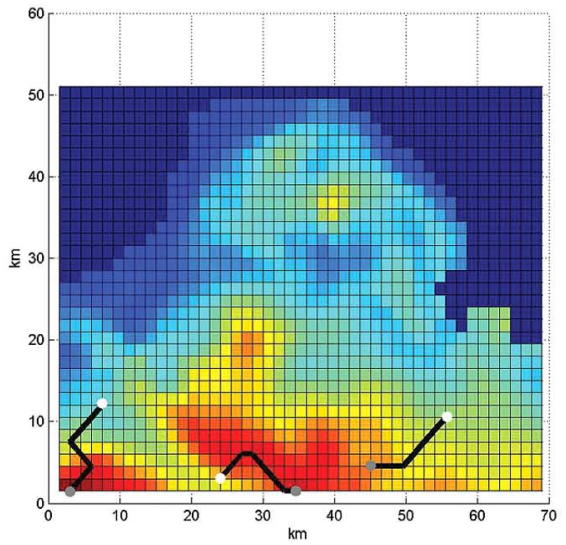

(c)

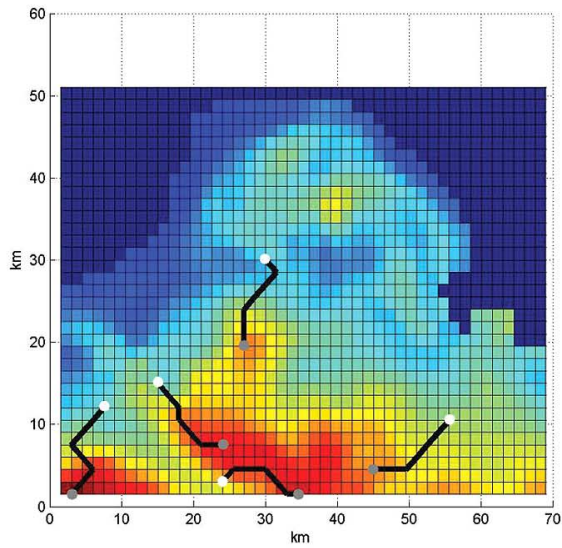

(e)

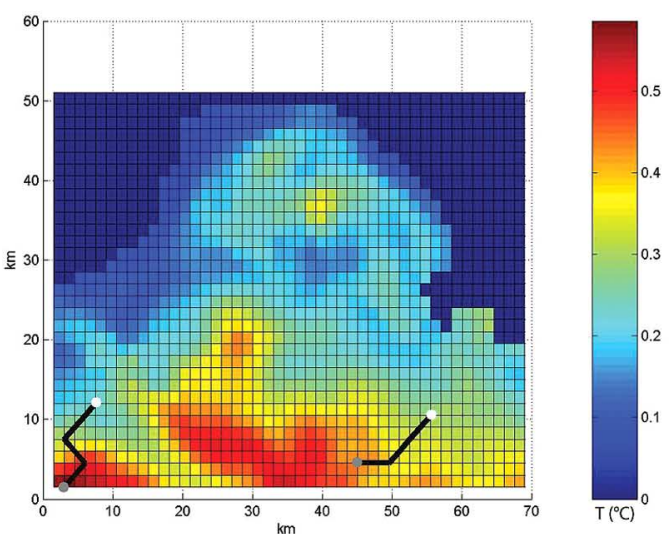

(b)
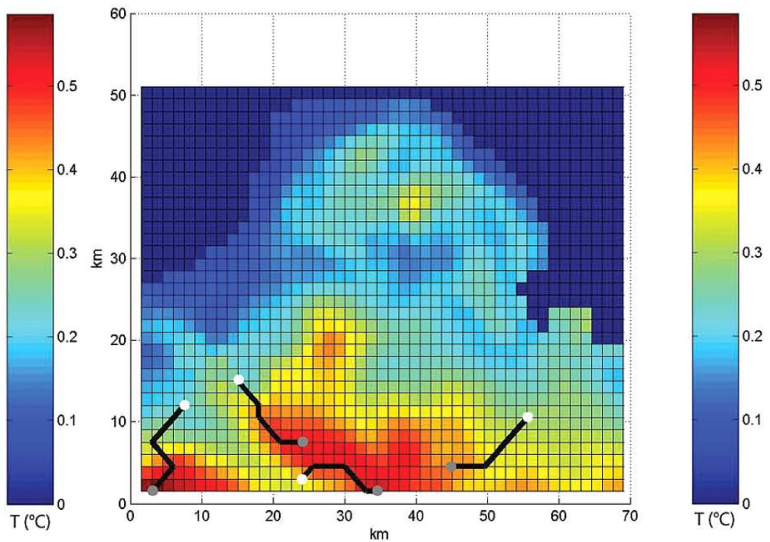

(d)
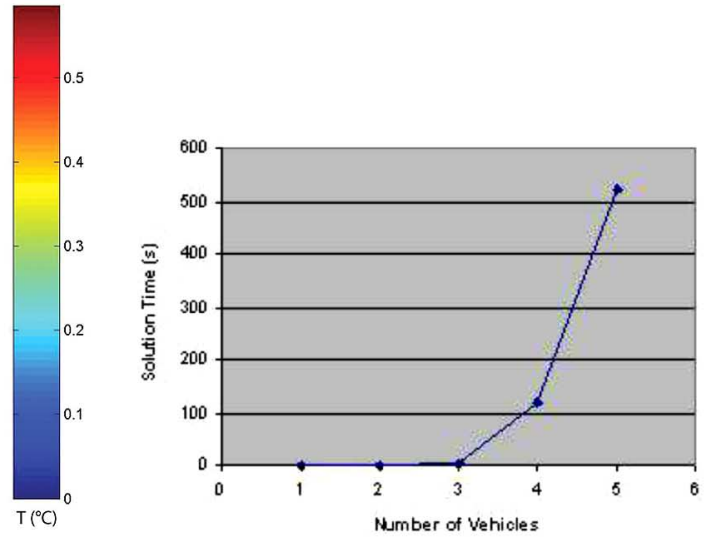

(f)

Fig. 7. (a) Results for the single-vehicle case. Starting coordinates: $x_{1}=7.5 \mathrm{~km}, y_{1}=8 \mathrm{~km}$, range ${ }_{1}=15 \mathrm{~km}$. (b) Results for the two-vehicle case. Starting coordinates: $x_{1}=7.5 \mathrm{~km}, y_{1}=8 \mathrm{~km}$, range ${ }_{1}=15 \mathrm{~km}$ and $x_{2}=55.5 \mathrm{~km}, y_{2}=10.5 \mathrm{~km}$, range ${ }_{2}=13 \mathrm{~km}$. (c) Results for the three-vehicle case. Starting coordinates: $x_{1}=7.5 \mathrm{~km}, y_{1}=8 \mathrm{~km}$, range ${ }_{1}=15 \mathrm{~km}$ and $x_{2}=55.5 \mathrm{~km}, y_{2}=10.5 \mathrm{~km}$, range ${ }_{2}=13 \mathrm{~km}$, and $x_{3}=24 \mathrm{~km}, y_{3}=3 \mathrm{~km}$, range ${ }_{3}=13.5 \mathrm{~km}$. (d) Results for the four-vehicle case. Starting coordinates: $x_{1}=7.5 \mathrm{~km}, y_{1}=8 \mathrm{~km}$, range ${ }_{1}=15 \mathrm{~km} ; x_{2}=55.5 \mathrm{~km}, y_{2}=10.5 \mathrm{~km}$, range ${ }_{2}=13 \mathrm{~km} ; x_{3}=$ $24 \mathrm{~km}, y_{3}=3 \mathrm{~km}$, range ${ }_{3}=13.5 \mathrm{~km} ; x_{4}=15 \mathrm{~km}, y_{4}=15 \mathrm{~km}$, range ${ }_{4}=13 \mathrm{~km}$. (e) Results for the five-vehicle case. Starting coordinates: $x_{1}=7.5 \mathrm{~km}, y_{1}=$ $8 \mathrm{~km}$, range ${ }_{1}=15 \mathrm{~km} ; x_{2}=55.5 \mathrm{~km}, y_{2}=10.5 \mathrm{~km}$, range ${ }_{2}=13 \mathrm{~km} ; x_{3}=24 \mathrm{~km}, y_{3}=3 \mathrm{~km}$, range ${ }_{3}=13.5 \mathrm{~km} ; x_{4}=15 \mathrm{~km}, y_{4}=15 \mathrm{~km}$, range ${ }_{4}=$ $13 \mathrm{~km} ; x_{5}=30 \mathrm{~km}, y_{5}=30 \mathrm{~km}$, range ${ }_{5}=13 \mathrm{~km}$. (f) Solution times as a function of number of vehicles in the fleet.

(26) are utilized in our formulation. The first case, where the defined proximity is set to $15 \mathrm{~km}$ takes $9 \mathrm{~s}$ to solve. When the proximity value is decreased to 9 , the solution time also decreases to $5.25 \mathrm{~s}$. The improvement is expected because tightening the constraint also shrinks the search space, resulting in strides in the solution time.
Another possible scenario is when the communication is via direct link in which case the AUVs do not need to stay near the ship throughout their mission, but must either park in some proximity of the ship or return to the ship at the end of their travel. This time, equations (27)-(29) are used. Fig. 8(c) and (d) presents the examples of the latter and former 


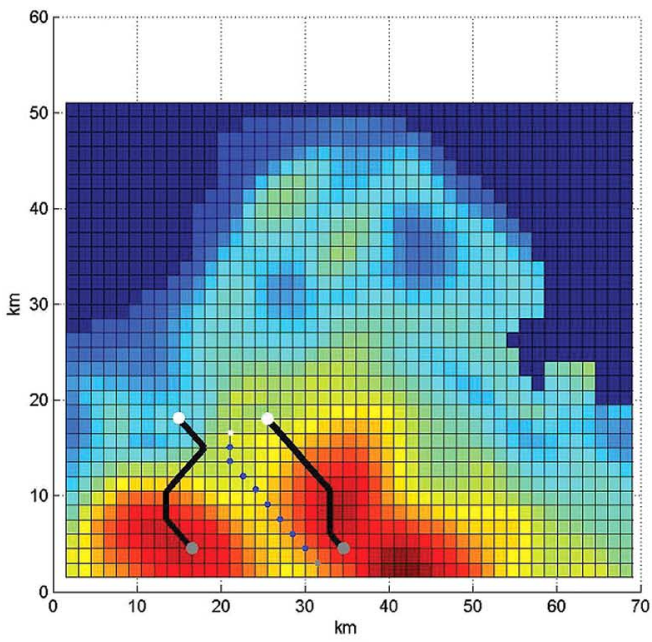

(a)

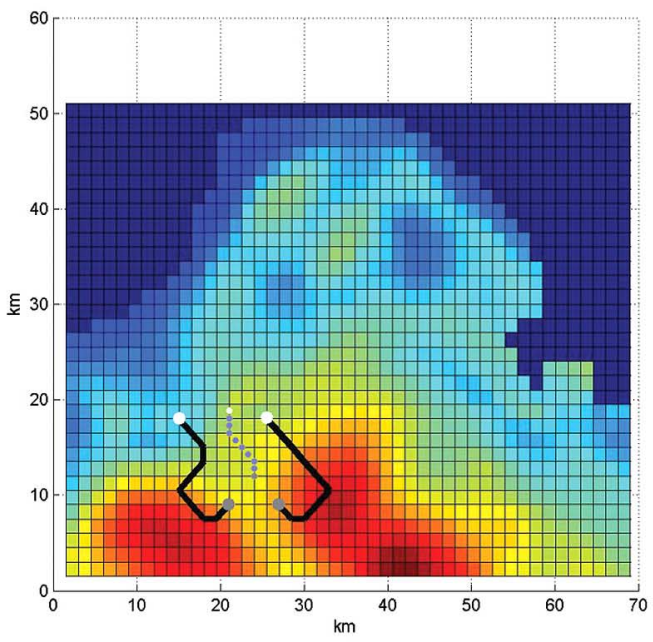

(c)
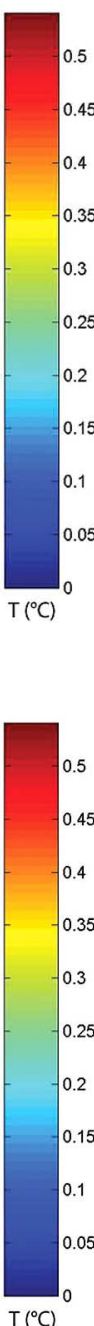

$\mathrm{T}\left({ }^{\circ} \mathrm{C}\right)$
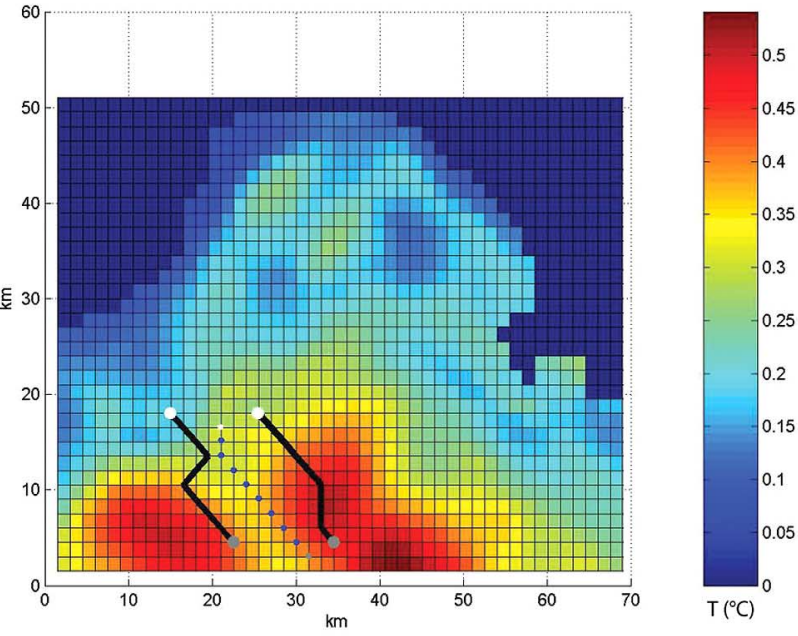

(b)

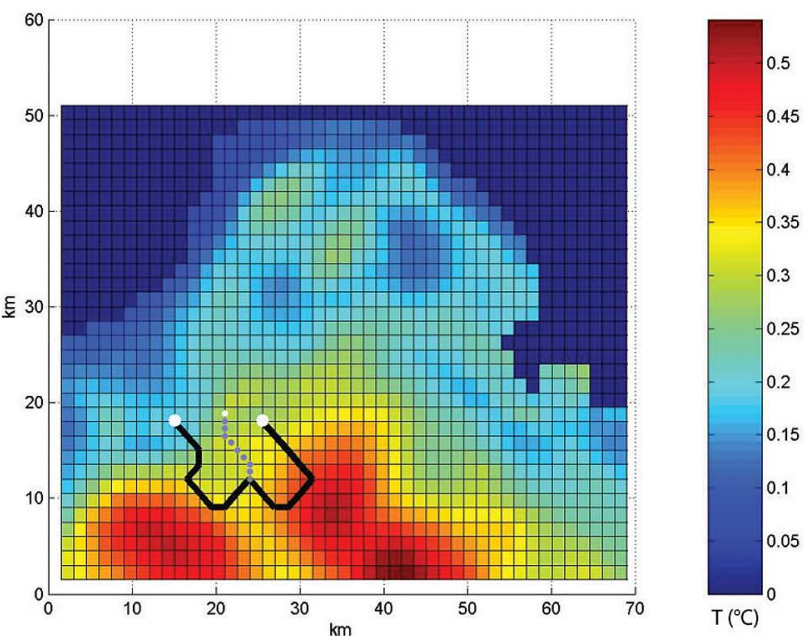

(d)

Fig. 8. (a) Results for two vehicles shadowed by a ship for the case where AUVs must be in 15-km vicinity of the ship. Ship path is shown with dotted line. Starting coordinates: $x_{1}=15 \mathrm{~km}, y_{1}=18 \mathrm{~km}$, range ${ }_{1}=19 \mathrm{~km} ; x_{2}=25.5 \mathrm{~km}, y_{2}=18 \mathrm{~km}$, range ${ }_{2}=18 \mathrm{~km}$. (b) Results for two vehicles shadowed by a ship for the case where AUVs must be in 9-km vicinity of the ship. Ship path is shown with dotted line. Starting coordinates: $x_{1}=15 \mathrm{~km}, y_{1}=18 \mathrm{~km}$, range ${ }_{1}=19 \mathrm{~km} ; x_{2}=25.5 \mathrm{~km}, y_{2}=18 \mathrm{~km}$, range ${ }_{2}=18 \mathrm{~km}$. (c) Results for two vehicles shadowed by a ship for the case where the end path points of AUVs must be in 3-km vicinity of the ship. Ship path is shown with dotted line. Starting coordinates: $x_{1}=15 \mathrm{~km}, y_{1}=18 \mathrm{~km}$, range ${ }_{1}=19 \mathrm{~km} ; x_{2}=25.5 \mathrm{~km}, y_{2}=$ $18 \mathrm{~km}$, range ${ }_{2}=18 \mathrm{~km}$. (d) Results for two vehicles shadowed by a ship for the case where the AUVs must return to the ship. Ship path is shown with dotted line. Starting coordinates: $x_{1}=15 \mathrm{~km}, y_{1}=18 \mathrm{~km}$, range ${ }_{1}=19 \mathrm{~km} ; x_{2}=25.5 \mathrm{~km}, y_{2}=18 \mathrm{~km}$, range ${ }_{2}=18 \mathrm{~km}$.

cases, respectively. The region of proximity is defined to be $3 \mathrm{~km}$ for Fig. 8(c). The solution time is $2.3 \mathrm{~s}$. For the case where AUVs need to return to the ship, the solution time is $2.14 \mathrm{~s}$.

\section{Time-Progressive Path PlanNing}

Up until this point, the sampling task have been assumed to take place in a single day without any pertinence to previous or following days. This is a perfectly fine scenario in rapid assessment in oceanography and it has been very successful for multiple uses at sea [7], [20]-[22], [55], [55]. However, a more sophisticated situation emerges when the sampling task has to be carried out over multiple days. In such time-evolving situations, the regions of high uncertainty are moving and transforming in shape as time progresses [4], [32] and the adaptive sampling fleet must adapt to the dynamic uncertainty field. In such scenarios, it is necessary to have some information exchange and coordination between the paths of the vehicles that are expected to be realized on consecutive days, so as to satisfy path optimality over both space and time.

A time dimension is introduced both for the primary and auxiliary variables as follows. A new index is added to every variable to represent the day they belong to. The new objective function is then a summation of rewards from all days under consideration. A key point in establishing the link between consecutive days and introducing time-progressive features is to define the relation between the end path point of vehicles on one day with the starting point on the following day. One option is to introduce the constraint that the starting point of the vehicle for a consecutive mission day should lie within a vicinity of the endpoint of the previous day. Another option is to impose the 
constraint that on consecutive mission days the vehicles should start their mission exactly at the end location of the previous day. This latter constraint can be defined as follows:

$$
\begin{aligned}
-\forall p \in[1, \ldots, P] \text { and } \forall d & \in[2, \ldots, D] \\
x_{p d 1} & =x_{p(d-1) N_{p}} \\
y_{p d 1} & =y_{p(d-1) N_{p}} .
\end{aligned}
$$

where $D$ stands for the total number of mission days.

To further exemplify the inclusion of time dimension, the objective function can be written as follows:

- Maximize

$$
\sum_{p=1}^{P} \sum_{d=1}^{D} \sum_{k=1}^{N_{p}} f_{p d k} .
$$

The reader is referred to [39] for the full formulation of the time-progressive case.

Assuming that AUVs have enough total range to complete sampling over the defined duration without any need to dock to get recharged and they continue their mission at the endpoint of the previous day, an example problem is solved whose results are presented in Fig. 9. This example also reveals capabilities of the proposed formulation to find time global optimal solutions. The number of path points chosen for both vehicles on both days is eight, which leads to a range of $15 \mathrm{~km}$ per day. If we assume the absence of any information link between the uncertainty data for day 1 and day 2, looking at Fig. 9(a), on day 1, the second vehicle, which starts its motion at $x=45 \mathrm{~km}$ and $y=30 \mathrm{~km}$, would have needed to be close to the small peak located around $x=40 \mathrm{~km}$ and $y=35 \mathrm{~km}$. With the two-day information available, vehicle 2 moves on day 1 such as shown in Fig. 9(a). Because there is a constrained connection between day 1 and day 2, vehicle 2 compromises on the total amount of rewards it can collect on day 1 and heads towards the high-uncertainty region that is predicted to form on day 2 around $x=35 \mathrm{~km}$ and $y=10 \mathrm{~km}$. Over the two days, this enables the maximization of total reward.

The above discussion can be easily extended to a 3-D case by simply adding an index for the $z$ coordinate to most of the variables involved in the formulation. This adds some new formulation variables related to the additional $z$ coordinate and modifies the right-hand sides of some of the inequalities.

\section{CONCLUSION AND DisCUSSIONS}

In this paper, we have addressed the problem of path planning of AUVs for adaptive sampling. We introduced an MILP-based formulation, which is capable of handling multiple-vehicle and multiple-day cases. Using MILP formulation techniques, it is possible to successfully model all the constraints needed for different problem scenarios. The strength of the MILP formulation makes future problem formulation extensions and modifications possible. This point was exemplified within the AOSN concept [46].

We first developed the details of our optimization formulation, including the objective function and a wide range of constraints. Once formulated, we implemented and solved the problem using the XPress-MP optimization suit from "Dash Optimization." We preferred to use the native optimization formulation environment from Dash Optimization called Mosel to
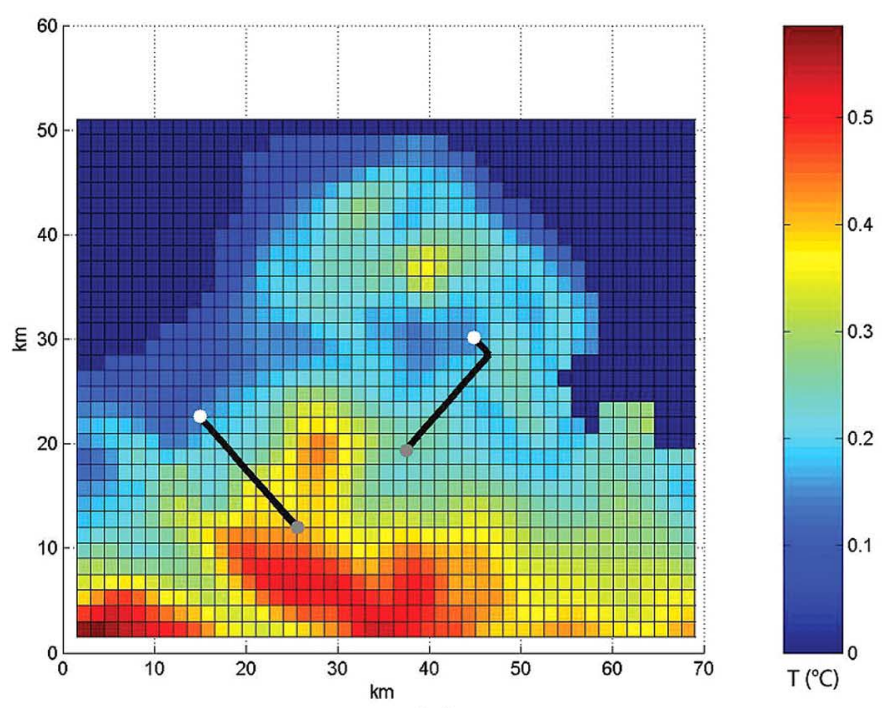

(a)

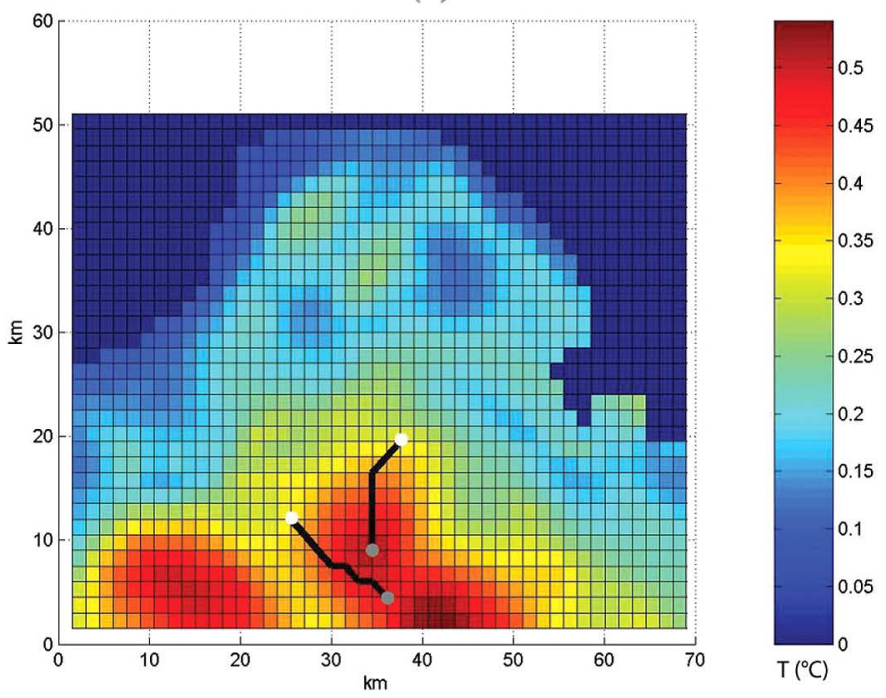

(b)

Fig. 9. (a) Results for the first day of a time-progressive case example. Starting coordinates: $x_{1}=15 \mathrm{~km}, y_{1}=22.5 \mathrm{~km}$, range ${ }_{1}=15 \mathrm{~km} ; x_{2}=45 \mathrm{~km}, y_{2}=$ $30 \mathrm{~km}$, range ${ }_{2}=15 \mathrm{~km}$. (b) Results for the second day of a time-progressive case example. Starting coordinates: $x_{1}=25.5 \mathrm{~km}, y_{1}=12 \mathrm{~km}$, range ${ }_{1}=$ $13.5 \mathrm{~km} ; x_{2}=37.5 \mathrm{~km}, y_{2}=19.5 \mathrm{~km}$, range ${ }_{2}=12 \mathrm{~km}$.

manage the formulation task. However, the formulation can be easily implemented with other optimization solver platforms. Using our new approach, we demonstrated a set of results for a wide range of scenarios using realistic ocean uncertainty fields. The effects of variations on the type of constraints, number of vehicles, and time dependence were studied and diverse sensitivity studies were carried out. In all cases, the results show that the method is capable of generating desired solutions within allotted time limits.

The problem we study is an NP hard problem. Therefore, as the problem size increases, the solution time increases exponentially. For the sizes, we considered that the solution time was short, especially in comparison to the time required to compute forecast ocean fields and their uncertainties. Our path-planning results were obtained on Pentium 4, 2.8-GHz computer with 1 GB of RAM. For larger problems, faster machines and 
grid/parallel computing are two options. The XPress-MP optimization suit we used already supports the parallel computing.

There remain many directions for future research. For example, the correlation between the measurement performed at one location and the immediate and future changes of uncertainty values around that location as a result of the collected data can be taken into account [22]. In future work, we are planning to establish the link between a measurement performed at a generated path point and its effect on the uncertainty field or fields involved in the problem, including ESSE technique into our optimization framework. Another line of research is the utilization of XML schemes [56] that control the parameters of our path-planning schemes and couple the optimization with the ocean modeling and data assimilation schemes.

The framework we supplied can also be extended to be utilized at low-level path planning where linearized vehicle dynamics and some waypoint information coming from high-level programming can be combined to smooth the path in an optimal manner. Another avenue of further research is the use of alternative solution techniques that can quickly generate suboptimal integer solutions and can warm-start the branch and bound algorithms. Candidate techniques include genetic algorithms and development of heuristics.

\section{ACKNOWLEDGMENT}

The authors would like to thank the whole Autonomous Ocean Sampling Network II (AOSN-II) team and colleagues for their real-time work in Monterey Bay, CA. They would also like to thank P. J. Haley, W. G. Leslie, A. R. Robinson, N. Leonard, H. Schmidt, D. Paley, and W. Cho for collaborations on ocean modeling and adaptive sampling. The authors also thank Prof. D. Pucci De Farias and Prof. J. Leonard for the valuable discussions.

\section{REFERENCES}

[1] M. Ehrendorfer, "Predicting the uncertainty of numerical weather forecasts: A review," Meteorologische Zeitschrift, vol. 4, pp. 47-183, 1997.

[2] P. F. J. Lermusiaux, "Data assimilation via error subspace statistical estimation. Part II: Middle Atlantic Bight shelfbreak front simulations and ESSE validation," Monthly Weather Rev., vol. 127, no. 7, pp. 1408-1432, 1999.

[3] C. H. Bishop and Z. Toth, "Ensemble transformation and adaptive observations," J. Atmos. Sci., vol. 56, pp. 1748-1765, 1999.

[4] P. F. J. Lermusiaux, C. S. Chiu, G. G. Gawarkiewicz, P. Abbot, A. R. Robinson, R. N. Miller, P. J. Haley, W. G. Leslie, S. J. Majumdar, A. Pang, and F. Lekien, "Quantifying uncertainities in ocean predictions," Oceanography, vol. 19, Special Issue on Advances in Computational Oceanography, no. 1, pp. 92-105, 2006, T. Paluszkiewicz and S. Harper, Eds..

[5] C. H. Bishop, B. J. Etherton, and S. J. Majumdar, "Adaptive sampling with the ensemble transform Kalman filter. Part I: Theoretical aspects," Monthly Weather Rev., vol. 129, pp. 420-436, 2001.

[6] P. F. J. Lermusiaux, C. Evangelinos, R. Tian, P. J. Haley, J. J. McCarthy, N. M. Patrikalakis, A. R. Robinson, and H. Schmidt, "Adaptive coupled physical and biogeochemical ocean predictions: A conceptual basis," in Lecture Notes in Computer Science, F. Darema, Ed. Berlin, Germany: Springer-Verlag, 2004, vol. 3038, pp. 685-692.

[7] A. R. Robinson and S. M. Glenn, "Adaptive sampling for ocean forecasting," Naval Res. Rev., vol. 51, no. 2, pp. 28-38, 1999.

[8] P. F. J. Lermusiaux, "Estimation and study of mesoscale variability in the Strait of Sicily," Dyn. Atmos. Oceans, vol. 29, pp. 255-303, 1999.
[9] T. M. Hamill and C. Snyder, "Using improved background-error covariances from an ensemble Kalman filter for adaptive observations," Monthly Weather Rev., vol. 130, pp. 1552-1572, 2002.

[10] P. F. J. Lermusiaux, P. J. Haley, and N. K. Yilmaz, "Environmental prediction, path planning and adaptive sampling: Sensing and modeling for efficient ocean monitoring, management and pollution control," Sea Technol., vol. 48, no. 9, pp. 35-38, 2007.

[11] P. F. J. Lermusiaux, P. Malanotte-Rizzoli, D. Stammer, J. Carton, J. Cummings, and A. M. Moore, "Progress and prospects of U.S. data assimilation in ocean research," Oceanography, vol. 19, Special Issue on Advances in Computational Oceanography, no. 1, pp. 172-183, 2006, T. Paluszkiewicz and S. Harper, Eds.

[12] T. N. Palmer, J. Gelaro, J. Barkmeijer, and R. Buizza, "Singular vectors, metrics, and adaptive observations," J. Atmos. Sci., vol. 55, pp. 633-653, 1998.

[13] R. Gelaro, R. H. Langland, G. D. Rohaly, and T. E. Rosmond, "An assessment of the singular vector approach to targeted observations using the FASTEX data set," Quart. J. Roy. Meteorol. Soc., vol. 125, pp. 3299-3328, 1999.

[14] R. Buizza and A. Montani, "Targeted observations using singular vectors," J. Atmos. Sci., vol. 56, pp. 1748-1765, 1999.

[15] T. Bergot, "Adaptive observations during FASTEX: A systematic survey of upstream flight," Quart. J. Roy. Meteorol. Soc., vol. 125, pp. 3271-3298, 1999.

[16] R. H. Langland and G. D. Rohaly, "Adjoint-based targeting of observations for FASTEX cyclones," in Proc. Amer. Meteorol. Soc. 7th Conf. Processes , 1996, pp. 369-371, Reprints.

[17] N. L. Baker and R. Daley, "Observation and background adjoint sensitivity in the adaptive observation-targeting problem," Quart. J. Roy. Meteorol. Soc., vol. 146, pp. 1431-1454, 2000.

[18] C. H. Bishop, C. A. Reynolds, and M. K. Tippett, "Optimization of the fixed global observing network in a simple model," J. Atmos. Sci., vol. 60, pp. 1471-1489, 2003.

[19] S. J. Majumdar, C. H. Bishop, and B. J. Etherton, "Adaptive sampling with the ensemble transform Kalman filter. Part II: Field program implementation," Monthly Weather Rev., vol. 130, pp. 1356-1369, 2002.

[20] P. F. J. Lermusiaux and A. R. Robinson, "Data assimilation via error subspace statistical estimation, Part I: Theory and schemes," Monthly Weather Rev., vol. 127, no. 8, pp. 1385-1407, 1999.

[21] P. F. J. Lermusiaux, "Evolving the subspace of the three-dimensional multiscale ocean variability: Massachusetts bay," J. Mar. Syst., vol. 29, Special Issue on Three-Dimensional Ocean Circulation: Lagrangian Measurements and Diagnostic Analyses, no. 1-4, pp. 385-422, 2001.

[22] P. F. J. Lermusiaux, C. K. R. T. Jones and K. Ide, Eds., "Adaptive sampling, adaptive data assimilation and adaptive modeling," Physica D, vol. 230, Special Issue on Mathematical Issues and Challenges in Data Assimilation for Geophysical Systems: Interdisciplinary Perspectives, pp. 172-196, 2007.

[23] E. Fiorelli, N. E. Leonard, P. Bhatta, D. Paley, R. Bachmayer, and D. M. Fratantoni, "Multi-AUV control and adaptive sampling in Monterey Bay," in Proc. Workshop Multiple AUV Operat., June 2004, pp. 134-147.

[24] D. J. Stilwell, B. E. Bishop, and C. A. Sylvester, "Redundant manipulator techniques for partially decentralized path planning and control of a platoon of autonomous vehicles," IEEE Trans. Syst. Man Cybern. B., Cybern., vol. 35, no. 4, pp. 842-848, Aug. 2005.

[25] C. J. Cannell and D. J. Stilwell, "A comparison of two approaches for adaptive sampling of environmental processes using autonomous underwater vehicles," in Proc. MTS/IEEE OCEANS Conf., Washington, DC, 2005, vol. 2, pp. 1514-1521.

[26] G. Laporte and S. Martello, "The selective travelling salesman problem," Discrete Appl. Math., vol. 26, pp. 193-207, 1990.

[27] E. Balas, "Prize collecting travelling salesman problem," Networks, vol. 19, pp. 621-636, 1989.

[28] B. L. Golden, L. Levy, and R. Vohra, "The orienteering problem," Naval Res. Logist., vol. 34, pp. 307-318, 1987.

[29] T. Schouwenaars, B. DeMoor, E. Feron, and J. How, Mixed Integer Programming for Safe Multi-Vehicle Cooperative Path Planning. Porto, Portugal: EEC, Sep. 2001, p. 6.

[30] A. Richards, J. Bellingham, M. Tillerson, and J. How, "Coordination and control of multiple UAVs," in Proc. AIAA Guid. Navigat. Control, Monterey, CA, Aug. 2002, vol. 5-8, pp. 2816-2822, paper AIAA2002-4588. 
[31] A. R. Robinson, "Forecasting and simulating coastal ocean processes and variabilities with the Harvard Ocean Prediction System," in Coastal Ocean Prediction, ser. AGU Coastal and Estuarine Studies. Washington, DC: American Geophysical Union, 1999, pp. $77-100$.

[32] P. F. J. Lermusiaux, "Uncertainty estimation and prediction for interdisciplinary ocean dynamics," J. Comput. Phys., vol. 217, Special Issue on Uncertainty Quantification, pp. 176-199, 2006, J. Glimm and G. Karniadakis, Eds.

[33] P. F. J. Lermusiaux, A. R. Robinson, P. J. Haley, and W. G. Leslie, “Advanced interdisciplinary data assimilation: Filtering and smoothing VIA error subspace statistical estimation," in Proc. MTS/IEEE OCEANSConf., vol. 2, pp. 795-802.

[34] D. Guo, C. Evangelinos, and N. M. Patrikalakis, "Flow feature extraction in oceanographic visualization," in Proc. Comput. Graph. Int. Conf., D. Cohen-Or, L. Jain, and N. Magnenat-Thalmann, Eds., Jun. 2004, pp. 162-173.

[35] D. Guo, "Automated feature extraction in oceanographic visualization," M.S. thesis, Mech. Eng. Dept., Massachusetts Inst. Technol., Cambridge, MA, Feb. 2004.

[36] K. Heaney, G. Gawarkiewicz, T. Duda, and P. Lermusiaux, "Non-linear optimization of autonomous undersea vehicle sampling strategies for oceanographic data-assimilation," J. Field Robot., vol. 24, Special Issue on Underwater Robotics, no. 6, pp. 437-448, 2007.

[37] E. M. L. Beale and J. A. Tomlin, "Special facilities in a general mathematical programming system for non-convex problems using ordered sets of variables," in Proc. 5th Int. Conf. Operat. Res., J. Lawrence, Ed., London, U.K., 1969, pp. 447-454.

[38] H. P. Williams, Model Building in Mathematical Programming, 4th ed. New York: Wiley, 1999, pp. 165-172.

[39] N. K. Yilmaz, "Path planning of autonomous underwater vehicles (AUVs) for adaptive sampling," Ph.D. dissertation, Mech. Eng. Dept., Massachusetts Inst. Technol., Cambridge, MA, Sep. 2005.

[40] D. Bertsimas and J. N. Tsitsiklis, Introduction to Linear Optimization. Belmont, MA: Athena Scientific, 1997.

[41] L. Freitag, M. Grand, C. von Alt, R. Stokey, and T. Austin, "A shallow water acoustic network for mine countermeasures operations with autonomous underwater vehicles," in Proc. Underwater Defense Technol. Conf., The Netherlands, 2005, pp. 1-20.

[42] H. Singh, J. G. Bellingham, F. H. S. Lemer, B. A. Moran, K. von der Heydt, and D. Yoerger, "Docking for an autonomous ocean sampling network," IEEE J. Ocean. Eng., vol. 26, no. 4, pp. 498-514, Oct. 2001.

[43] J. J. Leonard, A. A. Bennett, C. M. Smith, and H. J. S. Feder, "Autonomous underwater vehicle navigation," Dept. Ocean Eng., MIT Marine Robot. Lab., Cambridge, MA, Tech. Memorandum, Jan. 1998.

[44] M. S. L. Freitag, "Acoustic communications for regional undersea observatories," in Proc. Oceanology Int., London, U.K., Mar. 2002, paper AIAA-2004-6530.

[45] I. F. Akyildiz, D. Pompili, and T. Melodia, "Challenges for efficient communication in underwater acoustic sensor networks," C. ACM SIGBED Rev., vol. 1, pp. 3-8, 2004.

[46] T. B. Curtin, J. G. Bellingham, J. Catipovic, and D. Webb, "Autonomous oceanographic sampling networks," Oceanography, vol. 6, no. 3, pp. 86-94, 1993.

[47] H. Schmidt, "AREA: Adaptive rapid environmental assessment," in Impact of Littoral Environmental Variability on Acoustic Predictions and Sonar Performance, G. Pace and F. B. Jensen, Eds. Dordrecht, The Netherlands: Kluwer, 2006.

[48] D. Wang, P. F. J. Lermusiaux, P. J. Haley, D. Eickstedt, W. G. Leslie, and H. Schmidt, "Acoustically focused adaptive sampling and on-board routing for marine rapid environmental assessment," J. Mar. Syst., Special Issue on MREA and Coastal Processes: Challenges for Monitoring and Prediction, J. W. Book, M. Orlic, and M. Rixen, Eds., 2008, to be published.

[49] D. E. Chang, J. E. M. S. Shadden, and R. Olfati-Saber, "Collision avoidance for multiple agent systems," in Proc. Conf. Decision Control, 2003, pp. 539-543.

[50] P. Ogren and N. E. Leonard, "A convergent dynamic window approach to obstacle avoidance," IEEE Trans. Robot. Autom., vol. 21, no. 2, pp. 188-195, Apr. 2005.

[51] P. Ogren and N. E. Leonard, "Obstacle avoidance in formation," in Proc. IEEE Int. Conf. Robot. Autom., 2003, vol. 2, pp. 2492-2497.
[52] P. Ogren and N. E. Leonard, "A tractable convergent dynamic window approach to obstacle avoidance," in Proc. IEEE/RSJ Int. Conf. Intell. Robots Syst., 2002, vol. 21, pp. 188-195.

[53] P. J. Haley, Jr., P. F. J. Lermusiaux, A. R. Robinson, W. G. Leslie, O. Logoutov, G. Cossarini, X. S. Liang, P. Moreno, S. R. Ramp, J. D. Doyle, J. Bellingham, F. Chavez, and S. Johnston, "Real-time forecasting and re-analysis for the autonomous ocean sensing network-II (AOSN-II)," Deep Sea Res., Special Issue on AOSN-II, 2008, doi:10. 1016/j.dsr2.2008.08.010, to be published.

[54] S. R. Ramp, R. E. Davis, N. E. Leonard, I. Shulman, Y. Chao, A. R. Robinson, J. Marsden, P. F. J. Lermusiaux, D. Fratantoni, J. D. Paduan, F. Chavez, F. L. Bahr, S. Liang, X. Liang, W. Leslie, and Z. Li, "The autonomous ocean sensing network (AOSN) predictive skill experiment in the Monterey Bay," Deep Sea Res., Special Issue on AOSN-II, 2008, to be published.

[55] D. Wang, P. Lermusiaux, P. Haley, W. Leslie, and H. Schmidt, "Adaptive acoustical-environmental assessment for the focused acoustic field-05 at-sea exercise," in Proc. IEEE/MTS OCEANS Conf., Boston, MA, 2006, pp. 175-187.

[56] C. Evangelinos, P. F. J. Lermusiaux, S. Geiger, R. C. Chang, and N. M. Patrikalakis, "Web-enabled configuration and control of legacy codes: An application to ocean modeling," Ocean Model., pp. 197-220, 2006.

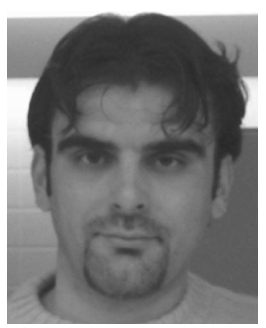

Namik Kemal Yilmaz received the B.S. degree in mechanical engineering with a minor in computer science from Middle East Technical University, Ankara, Turkey, in 1998 and the M.S. and Ph.D. degrees from the Mechanical Engineering Department, Massachusetts Institute of Technology, Cambridge, in 2001 and 2005, respectively.

His research interest are in path planning, optimization, design, and controls.

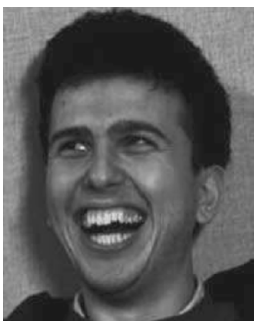

Constantinos Evangelinos received the B.A. degree (with honors) in mathematics from Cambridge University, Cambridge, U.K., in 1993 and the Sc.M. and $\mathrm{Ph} . \mathrm{D}$. degrees in applied mathematics from Brown University, Providence, RI, in 1994 and 1999, respectively.

Currently, he is a Research Scientist in the Earth, Atmospheric and Planetary Sciences Department, Massachusetts Institute of Technology, Cambridge, where he works on ocean state estimation. His current research interests are in computational methods for variational as well as sequential data assimilation, adjoint techniques and automatic differentiation, parallel/grid computing approaches and performance modeling applied to grand challenge applications in the ocean sciences.

Dr. Evangelinos is a member of the IEEE Computer Society and the Association for Computing Machinery (ACM).

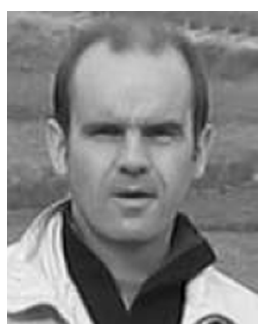

Pierre F. J. Lermusiaux received the B.-M.Eng. degrees (highest honors and Jury's congratulations) from Liege University, Liege, Belgium, in 1992 and the Ph.D. degree in engineering sciences from Harvard University, Cambridge, MA, in 1997.

Currently, he is an Associate Professor of Mechanical Engineering, Massachusetts Institute of Technology, Cambridge. His current research interests include physical and interdisciplinary ocean dynamics, from submesoscales to interannual scales. They involve physical-biogeochemical-acoustical ocean modeling, optimal estimation and data assimilation, uncertainty and error modeling, and the optimization of observing systems.

Dr. Lermusiaux has held Fulbright Foundation Fellowships, was awarded the Wallace Prize at Harvard in 1993, and presented the Ogilvie Young Investigator Lecture in Ocean Engineering at MIT in 1998. He is a member of the Association of Engineers of Liege University, Friends of the University of Liege, Royal Meteorological Society, American Geophysical Union, and Oceanography Society. 


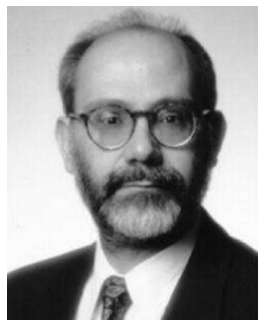

Nicholas Patrikalakis received the M.S. degree in naval architecture and mechanical engineering from the National Technical University of Athens, Athens, Greece, in 1977 and the Ph.D. degree in ocean engineering from Massachusetts Institute of Technology (MIT), Cambridge, in 1983.

Currently, he is the Kawasaki Professor of Engineering and Professor of Mechanical and Ocean Engineering at the Department of Mechanical Engineering, MIT. His current research focuses on marine robotics, shape similarity evaluation, and computer-aided design. Seventeen doctoral dissertations and 35 Master's theses have been completed under his direction. He has published over 140 papers and two textbooks and has edited 20 journal special issues or conference proceedings. He has received research funding from several federal agencies and industry in the United States and other countries.

Dr. Patrikalakis serves as editor or member of the editorial board of four international scientific journals. He has also served as program chair or chair of over ten major international conferences and has participated in the program committees of over 20 international conferences. 